\title{
WISE J072003.20-084651.2B is a Massive T Dwarf ${ }^{* \dagger}$
}

Trent J. Dupuy ${ }^{1}$ (1), Michael C. Liu ${ }^{2}$ (1), William M. J. Best ${ }^{3}$ (1), Andrew W. Mann ${ }^{4}$ (1), Michael A. Tucker ${ }^{2}$,, Zhoujian Zhang $^{2}$,

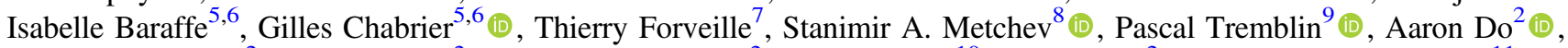
Anna V. Payne ${ }^{2}$, B. J. Shappee ${ }^{2}$ (D), Charlotte Z. Bond ${ }^{2}$, Sylvain Cetre ${ }^{10}$, Mark Chun ${ }^{2}$, Jacques-Robert Delorme ${ }^{11}$, Nemanja Jovanovic ${ }^{11}$, Scott Lilley ${ }^{10}$, Dimitri Mawet ${ }^{11}$ (i), Sam Ragland ${ }^{10}$, Ed Wetherell ${ }^{10}$, and Peter Wizinowich ${ }^{10}$

${ }_{1}^{1}$ Gemini Observatory, Northern Operations Center, 670 N. A'ohoku Place, Hilo, HI 96720, USA

${ }^{2}$ Institute for Astronomy, University of Hawaii, 2680 Woodlawn Drive, Honolulu, HI 96822, USA

${ }^{3}$ The University of Texas at Austin, Department of Astronomy, 2515 Speedway C1400, Austin, TX 78712, USA

${ }^{4}$ Department of Physics and Astronomy, University of North Carolina at Chapel Hill, Chapel Hill, NC 27599-3255, USA

${ }^{5}$ University of Exeter, Physics and Astronomy, EX4 4QL Exeter, UK

${ }^{6}$ Ècole Normale Supérieure, Lyon, CRAL (UMR CNRS 5574), Université de Lyon, France

${ }^{7}$ Université Grenoble Alpes, CNRS, IPAG, F-38000 Grenoble, France

${ }^{8}$ The University of Western Ontario, Department of Physics and Astronomy, 1151 Richmond Avenue, London, ON N6A 3K7, Canada

${ }^{9}$ Maison de la Simulation, CEA, CNRS, Univ. Paris-Sud, UVSQ, Université Paris-Saclay, F-91191 Gif-sur-Yvette, France

${ }^{10}$ W.M. Keck Observatory, 65-1120 Mamalahoa Hwy, Kamuela, HI, USA

${ }^{11}$ California Institute of Technology, Department of Astronomy, 1200 E. California Blvd., Pasadena, CA 91125, USA

Received 2019 June 7; revised 2019 August 11; accepted 2019 August 18; published 2019 October 10

\begin{abstract}
We present individual dynamical masses for the nearby M9.5+T5.5 binary WISE J072003.20-084651.2AB, a.k.a. Scholz's star. Combining high-precision Canada-France-Hawaii Telescope/WIRCam photocenter astrometry and Keck adaptive optics resolved imaging, we measure the first high-quality parallactic distance $\left(6.80_{-0.06}^{+0.05} \mathrm{pc}\right)$ and orbit $\left(8.06_{-0.25}^{+0.24} \mathrm{yr}\right.$ period) for this system composed of a low-mass star and brown dwarf. We find a moderately eccentric orbit $\left(e=0.240_{-0.010}^{+0.009}\right)$, incompatible with previous work based on less data, and dynamical masses of $99 \pm 6 M_{\text {Jup }}$ and $66 \pm 4 M_{\text {Jup }}$ for the two components. The primary mass is marginally inconsistent $(2.1 \sigma)$ with the empirical mass-magnitude-metallicity relation and models of main-sequence stars. The relatively high mass of the cold $\left(T_{\text {eff }}=1250 \pm 40 \mathrm{~K}\right)$ brown dwarf companion indicates an age older than a few gigayears, in accord with age estimates for the primary star, and is consistent with our recent estimate of $\approx 70 M_{\text {Jup }}$ for the stellar/substellar boundary among the field population. Our improved parallax and proper motion, as well as an orbit-corrected system velocity, improve the accuracy of the system's close encounter with the solar system by an order of magnitude. WISE J0720-0846AB passed within $68.7 \pm 2.0 \mathrm{kau}$ of the Sun $80.5 \pm 0.7 \mathrm{kyr}$ ago, passing through the outer Oort cloud where comets can have stable orbits.
\end{abstract}

Unified Astronomy Thesaurus concepts: Brown dwarfs (185); Astrometry (80); Visual binary stars (1777); Astrometric binary stars (79); T dwarfs (1679); M dwarf stars (982)

\section{Introduction}

While the nearest stars in the solar neighborhood are largely known, such stars residing in the Galactic plane may still remain to be discovered, in particular low-luminosity ones. As part of a search for such objects using the Two-micron All-sky Survey and Wide-field Infrared Survey Explorer (WISE), Scholz (2014) identified WISE J072003.20-084651.2 (hereinafter WISE J0720-0846) as a low-latitude $(b=+2.3)$ ultracool dwarf with an estimated spectral type of M9 \pm 1 and a parallactic distance of $6-8 \mathrm{pc}$ based on multicatalog photometry and astrometry. Follow-up by Ivanov et al. (2015) derived a spectral type of $\mathrm{LO} \pm 1$ based on optical and near-IR (NIR) spectroscopy and refined the parallax measurement, making it the third closest known L dwarf. They detected weak

\footnotetext{
* Data presented herein were obtained at the W. M. Keck Observatory, which is operated as a scientific partnership among the California Institute of Technology, the University of California, and the National Aeronautics and Space Administration. The Observatory was made possible by the generous financial support of the W. M. Keck Foundation.

$\dagger$ Based on data obtained with WIRCam, a joint project of CFHT, Taiwan, Korea, Canada, France, at the Canada-France-Hawaii Telescope, which is operated by the National Research Council of Canada, the Institute National des Sciences de l'Univers of the Centre National de la Recherche Scientifique of France, and the University of Hawaii.
}

$\mathrm{H} \alpha$ emission but not Li I absorption, consistent with an old age and a mass above the stellar/substellar boundary. Burgasser et al. (2015a) measured an optical spectral type of M9.5, found the spectrum to be consistent with solar metallicity, and observed variability (1\%-2\% amplitude) and flares (4\%-8\% amplitude) in TRAPPIST photometry. They estimated an age for the system of $0.5-5.0 \mathrm{Gyr}$ based on its 3D space motion indicating old-disk kinematics. Burgasser et al. (2015b) detected radio emission and a radial acceleration from Shane/Hamilton and Keck/NIRSPEC radial velocity (RV) monitoring. Aside from its proximity, WISE J0720-0846 is notable for being the star with the closest known past approach to the solar system, within $52_{-14}^{+23}$ kau only $70_{-10}^{+15} \mathrm{kya}^{12}$ (Mamajek et al. 2015), though other stars are likely to come even closer in the future (e.g., Gl 710 will pass within $\approx 20 \mathrm{kau}$ in $\approx 1$ Myr; Bailer-Jones 2018; de la Fuente Marcos \& de la Fuente Marcos 2018).

The binarity of WISE J0720-0846 has a convoluted history. Scholz (2014) speculated that the star could be a close binary system based on the discrepancy between his parallactic and photometric distance determinations, though the two quantities were formally consistent given the large

\footnotetext{
12 Thousand years ago.
} 
uncertainties. Ivanov et al. (2015) found no convincing evidence for binarity based on the integrated-light spectrum and a refined parallax, though they noted a possible indication of RV variability (at the $2 \sigma$ level) between measurements separated by three days. Burgasser et al. (2015a) suggested that the system harbors a T5-dwarf companion, based on visual identification of peculiarities in the $H$-band integrated-light spectrum consistent with weak methane absorption. (Quantitative analysis of the same spectrum by Bardalez Gagliuffi et al. (2014) did not flag this object as a candidate binary.) Possible confirmation of such a companion came from their Keck adaptive optics (AO) imaging showing a candidate source at 0 ". 14 separation, though our analysis here shows in fact this was a spurious detection. ${ }^{13}$ Follow-up AO imaging by Burgasser et al. (2015b) clearly showed a companion, with resolved NIR photometry leading to a revised NIR spectral type of T5.5 \pm 0.5 for the secondary. They also used their resolved imaging and integrated-light RVs to estimate an orbital period of $4.1_{-1.3}^{+2.7} \mathrm{yr}$ (our determination here is $8.06_{-0.25}^{+0.24} \mathrm{yr}$ ).

As part of our ongoing effort to measure dynamical masses for ultracool dwarfs (e.g., Liu et al. 2008; Dupuy \& Liu 2017), we have monitored WISE J0720-0846 with Keck AO and Canada-France-Hawaii Telescope (CFHT) wide-field astrometry for the past several years. There is only a handful of stars with T-dwarf companions at small separations amenable to orbit monitoring and thus dynamical mass determinations. WISE J0720-0846 offers the valuable opportunity to compare inferences about stellar (late-M dwarf) and substellar (T dwarf) properties that depend on age or composition, under the conservative assumption of the system being coeval and cocomposition. For instance, independent age measurements for the two components can be compared to assess the reliability of the age-determination methods, a.k.a., the isochrone test (e.g., Liu et al. 2010). In addition, the system straddles the stellar/ substellar mass boundary and thus provides an opportunity to help delineate this boundary among the field population. An ancillary product from such work is a high-precision proper motion and distance for the system (as it is not in Gaia DR2), which helps to understand its past dynamical interaction with the solar system.

\section{Observations}

\subsection{Keck/NIRC2 LGS AO}

We began monitoring WISE J0720-0846 on 2015 January 14 UT with NIRC2 and the laser guide star adaptive optics (LGS AO) system at the Keck II telescope (Bouchez et al. 2004; van Dam et al. 2006; Wizinowich et al. 2006). On the first two epochs (2015 January 15 and 2015 April 10 UT) we used a nearby star (USNO-B1.0 0812-0137391) that seemed it would provide better $\mathrm{AO}$ correction, but it turned out to be $1.2 \mathrm{mag}$ fainter than expected $(R \approx 17.6 \mathrm{mag})$. At all other epochs we used the science target itself as the tip-tilt reference star, even though its optical faintness can make acquisition challenging. However, the tip-tilt sensor is very red sensitive, so it detected counts equivalent to a star of $\approx 14.8$ mag in spite of this target's actual $R$-band magnitude of 16.9 mag (Monet et al. 2003). At all epochs, we obtained data using NIRC2's medium-band filter centered on the $H$-band flux peak seen in

\footnotetext{
${ }^{13}$ Deacon et al. (2017) reported a possible detection of binarity from analyzing the ellipticity of seeing-limited Pan-STARRS 1 images obtained during $\approx 2010-2014$, though they did not consider the result to be reliable.
}

T dwarfs $\left(\mathrm{CH}_{4 s} ; \lambda_{C}=1.592 \mu \mathrm{m}\right.$ and $\left.\Delta \lambda=0.126 \mu \mathrm{m}\right)$. This filter offered the best compromise between the quality of the $\mathrm{AO}$ correction (better at longer wavelengths) and the signal-tonoise ratio $(\mathrm{S} / \mathrm{N})$ of the companion (better at bluer NIR wavelengths). Observing in a medium-band filter also mitigates the influence of differential chromatic refraction (DCR) that would otherwise introduce systematic offsets in our relative astrometry given the very different spectra of the two components. At some epochs we also obtained data in standard Maunakea Observatories (MKO) filters (Simons \& Tokunaga 2002; Tokunaga et al. 2002) for the purposes of measuring relative photometry.

Figure 1 shows images from every epoch of our monitoring observations. At our first epoch, the companion was to the southwest and moving outward to wider separations. By 2015 September 23 UT, the companion had already started moving inward. Then, by 2016 October 11 UT, the companion was not resolved even though the image quality was comparable to or better than previous data sets. As we see later in our analysis, these nondetections are consistent with our derived orbit. A year later, the companion was recovered for the first time to the northeast at a separation of 0 .! 19 , and it has subsequently moved outward to its widest separation yet of 0 "! 38 .

At the epochs where the companion is resolved, we measured binary parameters (separation, PA, flux ratio) using similar methods as in our previous work (e.g., Liu et al. 2008; Dupuy et al. 2010). We fit an analytic, three-component Gaussian model to each point source when they are spatially blended, and when they are better separated we perform pointspread function (PSF) fitting using StarFinder (Diolaiti et al. $2000)$. We then convert the measured $(x, y)$ positions into sky coordinates using the same methods as described in Dupuy et al. (2016) and Dupuy \& Liu (2017), with the only difference being that we reversed the sign of the PA offsets of 0.252 and 0.262 in the Yelda et al. (2010) and Service et al. (2016) calibrations, respectively, as found by Bowler et al. (2018). At several recent epochs where the binary separation is rather wide $\left(\gtrsim 0\right.$ !' 3 ), the $4 \times 10^{-4}$ uncertainty in the pixel scale of NIRC2 is the dominant error term for our separation measurements. In most other cases, the rms of our dithered measurements dominates.

Table 1 lists all our derived binary parameters, where each quoted error is the rms of measurements from individual images. For separations and PAs, the systematic uncertainties in the astrometric calibration, e.g., 0.004 mas pixel $^{-1}$ and 0.020 for the Service et al. (2016) calibration, have been added in quadrature to the rms values. To assess the accuracy of our relative photometry, we examined the best $\mathrm{CH}_{4 s}$ data sets and found an rms of $0.04 \mathrm{mag}$ in $\Delta \mathrm{CH}_{4 s}$ values. We adopt this as a systematic noise floor for all of our relative photometry, most likely due to the limitations of our PSF modeling given that variability at this amplitude in the $H$ band is relatively uncommon (e.g., Metchev et al. 2015).

\subsection{Keck/NIRC2 PyWFS AO}

In addition to observations made using the facility LGS AO system, we also obtained $K$ - and $L^{\prime}$-band imaging with the newly installed infrared pyramid wavefront sensor (PyWFS) on Keck II. The PyWFS will be part of the Keck Planet Imager and Characterizer (Mawet et al. 2018), an instrument optimized for high-contrast observations of faint red objects. The design and laboratory testing of the PyWFS is detailed in 

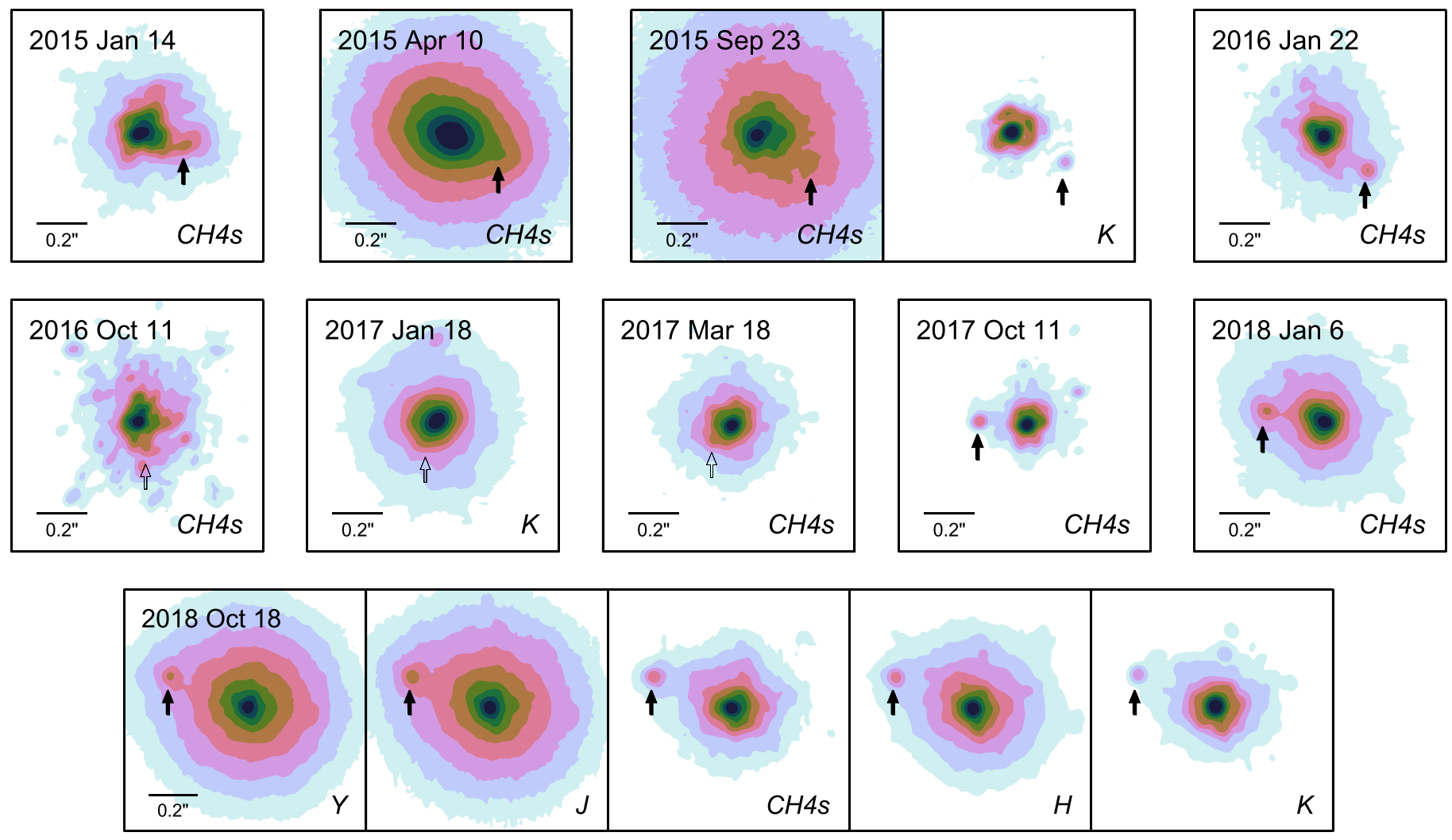

Figure 1. Contour plots of typical individual exposures from our Keck LGS AO data, with levels drawn in logarithmic intervals from unity down to $0.5 \%$ of the peak flux in each image. The images are all $1{ }^{\prime \prime} 0$ across and have been rotated so that north is up. An arrow indicates the position of the companion at each epoch, where unfilled arrows indicate the predictions from our orbit fit at epochs where the companion is not resolved (2016 October 11 UT to 2017 March 18 UT).

Table 1

Relative Astrometry from Keck/NIRC2 Adaptive Optics Imaging

\begin{tabular}{|c|c|c|c|c|c|c|c|c|c|c|}
\hline $\begin{array}{l}\text { Date } \\
\text { (UT) }\end{array}$ & $\begin{array}{l}\text { Separation } \\
\text { (mas) }\end{array}$ & $\begin{array}{l}\text { PA } \\
\left({ }^{\circ}\right)\end{array}$ & $\begin{array}{c}\Delta m \\
(\mathrm{mag})\end{array}$ & Filter & $\begin{array}{c}N_{\text {coadd }} \\
\times t_{\exp }(\mathrm{s})\end{array}$ & $N_{\exp }$ & Airmass & $\begin{array}{l}\text { Strehl } \\
\text { Ratio }\end{array}$ & $\begin{array}{c}\text { FWHM } \\
\text { (mas) }\end{array}$ & Notes \\
\hline 2015 Jan 14 & $188 \pm 5$ & $256.0 \pm 0.7$ & $2.87 \pm 0.10$ & $\mathrm{CH}_{4 s}$ & $30 \times 1.0$ & 11 & 1.14 & $0.084 \pm 0.015$ & $72 \pm 6$ & * \\
\hline 2015 Apr 10 & $225 \pm 9$ & $252.6 \pm 1.5$ & $3.9 \pm 0.5$ & $\mathrm{CH}_{4 s}$ & $30 \times 1.0$ & 9 & 1.45 & $0.025 \pm 0.005$ & $143 \pm 17$ & * \\
\hline 2015 Sep 23 & $239 \pm 6$ & $238.2 \pm 1.1$ & $2.85 \pm 0.11$ & $\mathrm{CH}_{4 s}$ & $50 \times 1.0$ & 6 & 1.43 & $0.046 \pm 0.020$ & $63 \pm 11$ & * \\
\hline 2015 Sep 23 & $241.7 \pm 1.1$ & $239.87 \pm 0.24$ & $3.880 \pm 0.020$ & $K$ & $10 \times 0.36$ & 2 & 1.46 & $0.326 \pm 0.029$ & $53 \pm 0$ & UX \\
\hline 2016 Jan 22 & $222.8 \pm 1.0$ & $232.12 \pm 0.17$ & $3.30 \pm 0.15$ & $\mathrm{CH}_{4 s}$ & $20 \times 1.0$ & 11 & 1.42 & $0.110 \pm 0.021$ & $53 \pm 3$ & * \\
\hline 2017 Oct 11 & $188.5 \pm 0.8$ & $85.77 \pm 0.06$ & $2.945 \pm 0.013$ & $\mathrm{CH}_{4 s}$ & $20 \times 1.0$ & 10 & 1.19 & $0.197 \pm 0.008$ & $46 \pm 2$ & * \\
\hline 2018 Jan 6 & $232.8 \pm 1.1$ & $79.32 \pm 0.06$ & $3.017 \pm 0.021$ & $\mathrm{CH}_{4 s}$ & $20 \times 1.0$ & 11 & 1.14 & $0.081 \pm 0.014$ & $60 \pm 4$ & * \\
\hline 2018 Oct 18 & $346.1 \pm 1.4$ & $67.937 \pm 0.022$ & $2.910 \pm 0.009$ & $Y$ & $6 \times 20.0$ & 3 & 1.25 & $0.077 \pm 0.005$ & $61 \pm 2$ & UX \\
\hline 2018 Oct 18 & $345.8 \pm 1.4$ & $67.864 \pm 0.027$ & $2.581 \pm 0.012$ & $J$ & $20 \times 1.0$ & 4 & 1.27 & $0.030 \pm 0.006$ & $61 \pm 5$ & UX \\
\hline 2018 Oct 18 & $346.1 \pm 1.6$ & $67.78 \pm 0.06$ & $2.971 \pm 0.008$ & $\mathrm{CH}_{4 s}$ & $20 \times 1.0$ & 5 & 1.32 & $0.076 \pm 0.019$ & $60 \pm 6$ & * \\
\hline 2018 Oct 18 & $346.5 \pm 1.4$ & $67.75 \pm 0.04$ & $3.201 \pm 0.019$ & $H$ & $20 \times 1.0$ & 4 & 1.28 & $0.086 \pm 0.011$ & $57.8 \pm 1.5$ & UX \\
\hline 2018 Oct 18 & $346.4 \pm 1.4$ & $67.61 \pm 0.03$ & $3.789 \pm 0.015$ & $K$ & $20 \times 1.0$ & 8 & 1.29 & $0.214 \pm 0.025$ & $62.4 \pm 1.4$ & $\mathrm{X}$ \\
\hline 2019 Apr 21 & $386.9 \pm 1.7^{\mathrm{a}}$ & $63.44 \pm 0.09^{\mathrm{a}}$ & $3.867 \pm 0.013$ & K & $3 \times 1.0$ & 4 & 1.55 & $0.266 \pm 0.074$ & $58 \pm 3$ & PUX \\
\hline 2019 Apr 21 & $381.2 \pm 2.2^{\mathrm{a}}$ & $63.53 \pm 0.27^{\mathrm{a}}$ & $3.01 \pm 0.03$ & $L^{\prime}$ & $20 \times 0.2$ & 43 & 1.61 & $0.687 \pm 0.055$ & $85.8 \pm 0.9$ & PX \\
\hline
\end{tabular}

Notes. $\left({ }^{*}\right)$ used in orbit fit; (P) pyramid wavefront sensor observations; (U) rms errors likely underestimated because $N_{\exp } \leqslant 4$; (X) not used in orbit fit.

${ }^{a}$ Astrometric calibration for the pyramid wavefront sensor system used with NIRC2 is not yet measured. We use the Service et al. (2016) solution to quote representative numbers for separation and PA, but these should not be used for science. The orbit fit we derive in Section 3 predicts a separation of $386 \pm 4$ mas and PA of $63.28 \pm 0.16$ at this epoch.

Bond et al. (2018). The instrument is currently being commissioned, and the data presented here were taken on the first sharedrisk science night (2019 April 21 UT). The wavefront sensing was done in the $H$ band $(\lambda=1.65 \mu \mathrm{m})$, with a pyramid modulation of $3 \lambda / D$, thus enabling high-Strehl ratio NGS AO observations of red objects that are faint in the optical.

Table 1 includes astrometry measured from our PyWFS AO imaging, which is displayed in Figure 2. The configuration of
NIRC2 with the PyWFS differs from the facility AO configuration, as an additional dichroic sends $J$ - and $H$-band light to the PyWFS, with the rest of the infrared light transmitted to NIRC2. Precise astrometric calibration of NIRC2 in this configuration is a work in progress, so PyWFS astrometry should not yet be used for science. We note however that if we simply adopt the Service et al. (2016) calibration, then the astrometry is in good agreement with our 
Table 2

CFHT/WIRCam Astrometry of WISE J0720-0846AB in Integrated Light

\begin{tabular}{|c|c|c|c|c|c|c|c|}
\hline \multicolumn{2}{|c|}{ Observation Date } & \multirow{2}{*}{$\begin{array}{l}\text { R.A. } \\
\text { (deg) }\end{array}$} & \multirow{2}{*}{$\begin{array}{l}\text { Decl. } \\
\text { (deg) }\end{array}$} & \multirow{2}{*}{$\begin{array}{l}\sigma_{\text {R.A. }} \\
\text { (mas) }\end{array}$} & \multirow{2}{*}{$\begin{array}{l}\sigma_{\text {Decl. }} \\
(\mathrm{mas})\end{array}$} & \multirow{2}{*}{ Airmass } & \multirow{2}{*}{$\begin{array}{l}\text { Seeing } \\
(\operatorname{arcsec})\end{array}$} \\
\hline (UT) & (MJD) & & & & & & \\
\hline 2015 Feb 8 & 57061.3162 & 110.01352251 & -08.78093445 & 3.5 & 5.2 & 1.175 & 0.60 \\
\hline 2015 Dec 23 & 57379.4871 & 110.01354626 & -08.78095899 & 4.1 & 4.5 & 1.140 & 0.71 \\
\hline 2016 Sep 12 & 57643.6386 & 110.01354930 & -08.78096045 & 7.3 & 9.6 & 1.613 & 0.58 \\
\hline 2017 Mar 15 & 57827.2925 & 110.01345537 & -08.78099102 & 2.4 & 4.2 & 1.170 & 0.57 \\
\hline 2017 Dec 5 & 58092.5417 & 110.01349091 & -08.78104046 & 3.3 & 7.2 & 1.142 & 0.67 \\
\hline 2018 Nov 21 & 58443.5650 & 110.01347304 & -08.78107995 & 1.5 & 2.5 & 1.140 & 0.62 \\
\hline 2019 Mar 23 & 58565.2709 & 110.01339713 & -08.78107819 & 2.2 & 2.9 & 1.168 & 0.51 \\
\hline
\end{tabular}

Note. The quoted uncertainties correspond to relative, not absolute, astrometric errors.

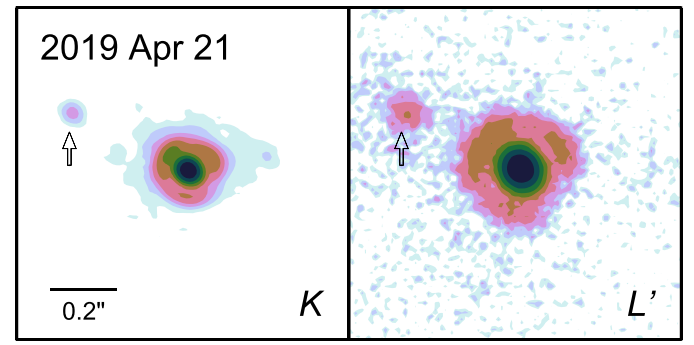

Figure 2. Contour plots of typical individual exposures from Keck/NIRC2 obtained with PyWFS AO. As in Figure 1, levels are drawn in logarithmic intervals from unity to $0.5 \%$ of the peak flux in each band, and the images are all 1"! 0 across and have been rotated so that north is up. An arrow indicates the position of the companion, predicted from the orbit fit that does not use these data.

orbit determination from Section 3 that predicts a separation of $386 \pm 4$ mas and PA of $63^{\circ} .28 \pm 0.16$ at the PyWFS epoch.

\section{3. $C F H T / W I R C a m$}

We first began monitoring WISE J0720-0846 at the CFHT on 2015 February 8 UT as part of the Hawaii Infrared Parallax Program (Dupuy \& Liu 2012), and since then obtained most of our data from our ongoing Large Program, the CFHT Infrared Parallax Program. The facility infrared camera WIRCam (Puget et al. 2004) provides wide-field, seeing-limited imaging. For WISE J0720-0846, we obtained data using a narrow-band filter ( $0.032 \mu \mathrm{m}$ bandwidth) in the $K$ band. We refer to this filter as $K_{\mathrm{H} 2}$ band because it is centered at $2.122 \mu \mathrm{m}$, the wavelength of the $\mathrm{H}_{2}$ 1-0 S(1) line. We use this filter for our brighter targets that risk saturation in wider bandpasses, and a side benefit of using such a narrow-band filter is that it renders DCR negligible.

We typically obtained 18 images per epoch with exposure times of $5 \mathrm{~s}$ that resulted in $\mathrm{S} / \mathrm{N} \approx 600$ on the target. We measured $(x, y)$ positions of the target and 145 reference stars in the field using SExtractor (Bertin \& Arnouts 1996, using windowed Gaussian parameters) and converted these to precision multi-epoch relative astrometry using a custom pipeline described in detail in our previous work (Dupuy \& Liu 2012; Liu et al. 2016). The absolute calibration of the linear terms of our astrometric solution was derived by matching low proper motion

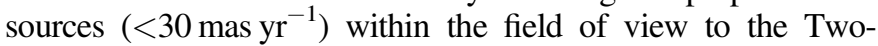
micron All-sky Survey (2MASS) point source catalog (Cutri et al. 2003). The resulting astrometry for WISE J0720-0846 in integrated light is given in Table 2. To convert our relative parallax and proper motion to an absolute frame, we used the mean and standard deviation of the simulated Galaxy population from the Besançon model (Robin et al. 2003).

\subsection{UH $2.2 \mathrm{~m} / \mathrm{SNIFS}$}

We obtained an optical spectrum of WISE J0720-0846 with the SuperNova Integral Field Spectrograph (SNIFS; Aldering et al. 2002; Lantz et al. 2004) on the University of Hawai'i 2.2 $\mathrm{m}$ telescope on Maunakea on 2018 October 22 UT. SNIFS provides simultaneous coverage from 3200 to $9700 \AA$ at a resolution of $R \simeq 1200$, and the total exposure time of our observation was $3600 \mathrm{~s}$. Details of our SNIFS reduction can be found in Bacon et al. (2001) and Gaidos et al. (2014), which we briefly summarize here. The pipeline detailed in Bacon et al. (2001) performed dark, bias, and flat-field corrections, cleaned the data of bad pixels and cosmic rays, then fit and extracted the integral field unit spaxels into a 1D spectrum. The Gaidos et al. (2014) reduction takes the 1D spectrum and performs flux calibration and telluric correction based on white dwarf standards taken throughout the night and a model of the atmosphere above Maunakea (Buton et al. 2013).

\subsection{IRTF/SpeX}

We obtained an NIR spectrum of WISE J0720-0846 with the NASA Infrared Telescope Facility (IRTF). Our observations were taken on 2018 October 23 UT with clear skies and seeing of $\approx 0$." 9 . We used the facility spectrograph SpeX (Rayner et al. 2003 ) in the short-wavelength cross-dispersed (SXD) mode with the $0 . .3 \times 15^{\prime \prime}$ slit $(R \approx 2000)$ aligned with the parallactic angle (327.49). We took six exposures of $120 \mathrm{~s}$ each in a standard ABBA pattern to achieve $>100 \mathrm{~S} / \mathrm{N}$ per pixel in both $H$ and $K$ bands, sufficient for measuring precise metallicities of late-M dwarfs (e.g., Mann et al. 2014). We observed the A0V standard star HD 48481 within 20 minutes and 0.01 airmass of the science target for telluric correction. We reduced the SXD spectra in a standard fashion using version 4.1 of the Spextool software package (Cushing et al. 2004).

\section{Orbit, Parallax, and Dynamical Masses}

We combined our Keck LGS AO relative astrometry with our integrated-light astrometry from CFHT/WIRCam in a single analysis fitting the orbit, parallax, and proper motion. 

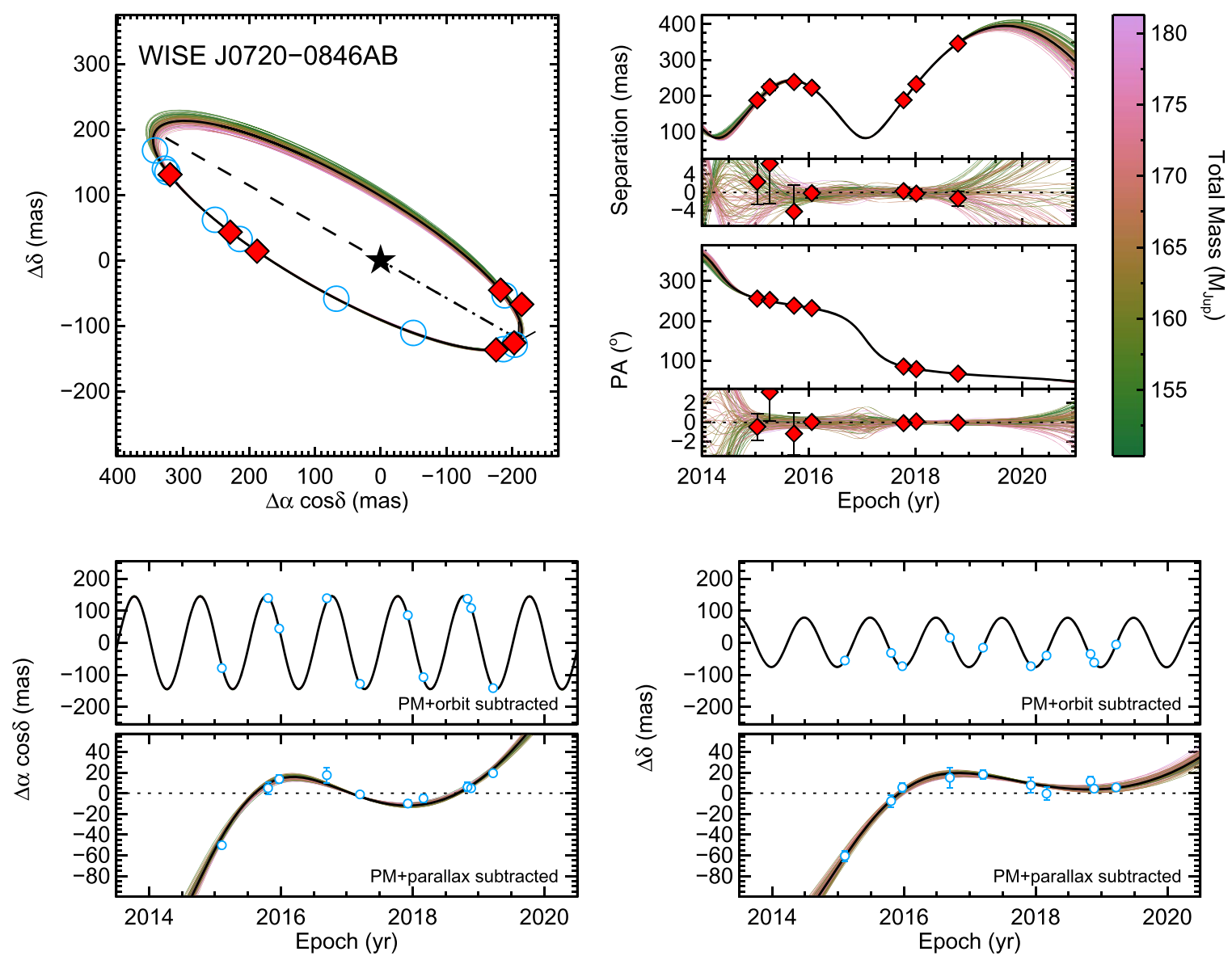

Figure 3. Our astrometry and orbit determination for WISE J0720-0846AB. In all panels, the best-fit orbit is a thick black line, and 100 orbit solutions drawn randomly from our MCMC posterior are thin lines colored according to the dynamical total mass (color bar in top right panel). Top left: relative astrometry from Keck LGS AO imaging (red diamonds). The times corresponding to the observation epochs with CFHT/WIRCam are marked by open blue circles. The line of nodes is indicated by a dashed line, and a dotted line connects the primary star to the point along the orbit corresponding to periastron passage (almost exactly overlapping with the southwest node here). Top right: relative astrometry as a function of time with the lower subpanels showing residuals from the best-fit orbit. Bottom: integratedlight astrometry from CFHT/WIRCam as a function of time. Upper subpanels show the parallax curve that remains after subtracting proper motion and orbital motion (errors are plotted but too small to be visible). Lower subpanels show the orbital motion that remains after subtracting proper motion and parallax. This is for display purposes only, as our analysis jointly fits all three (proper motion, parallax, and orbital motion) simultaneously.

We did not use the multibandpass averaged relative astrometry from Burgasser et al. (2015b) in our analysis, as that epoch (2015 January 11 UT) is contemporaneous with our first data (2015 January 14 UT).

Our approach is very similar to our past work (Dupuy et al. 2015; Dupuy \& Liu 2017). Six of the 13 parameters are shared between the resolved and integrated-light data, all relating to the orbit: period $(P)$, eccentricity $(e)$ and argument of periastron ( $\omega$ ) parameterized as $\sqrt{e} \sin \omega$ and $\sqrt{e} \cos \omega$ for the fit in order to accommodate near-circular orbits, inclination (i), P.A. of the ascending node $(\Omega)$, and mean longitude at the reference epoch $\left(\lambda_{\text {ref }}\right)$. The reference epoch $\left(t_{\text {ref }}\right)$ is defined to be 2010 January 1 00:00 UT (2455197.5 JD). There are two parameters for the size of the orbit. One is the total semimajor axis $(a)$ in angular units for the resolved orbit. The photocenter orbit size $\left(a_{\text {phot }}\right)$ is represented in our fit by the ratio $a_{\text {phot }} / a$. The five remaining parameters are all related to the CFHT astrometry: relative parallax $\left(\varpi_{\text {rel }}\right)$, proper motion $\left(\mu_{\text {rel }}\right)$ in R.A. and decl., and the R.A. and decl. at the reference epoch $t_{\text {ref. The only }}$ parameters without uniform priors were $P$ and $a$ (log-flat), $i$ (random viewing angles, i.e., sin $i$ ), and an approximately uniform space density prior $\left(\varpi_{\text {rel }}^{-4}\right)$.
We use the parallel-tempering Markov chain Monte Carlo (PTMCMC) ensemble sampler in emcee v2.1.0 (Foreman-Mackey et al. 2013) that is based on the algorithm described by Earl \& Deem (2005). Our results are based on the coldest of 30 chains, where the hottest chain effectively samples all of the allowed parameter space. We use 100 walkers to sample our thirteenparameter model over $8 \times 10^{4}$ steps. The initial state of the MCMC is a random, uniform draw over all of parameter space for bounded parameters $\left(e, \omega, \Omega, i, \lambda_{\text {ref }} ; 2 \mathrm{yr}<P<2000 \mathrm{yr}\right.$; 0 ". $01<\log \quad a<1$." $\left.0 ; \quad-1<a_{\text {phot }} / a<1\right) ; \quad$ Gaussian draw of \pm 100 mas around the least-squares fit of the reference epoch R.A. and decl.; $\pm 30 \%$ around the least-squares fit of the relative proper motion; and $\pm 20 \%$ around the least-squares fit of the relative parallax. After these wide ranging initial states, the PTMCMC converged quickly to a tightly clustered set of orbital parameters. We excluded the first $75 \%$ of the chain as burn-in, where the last portion that was kept after we verified that it had stabilized in the mean and rms (among walkers) for each parameter.

Our data is shown alongside the PT-MCMC orbit posterior in Figure 3. The marginalized posteriors of all of our fitted parameters, as well as some key parameters (like $e$ and $\omega$ ) computed from them, are shown in Figure 4 and summarized in 

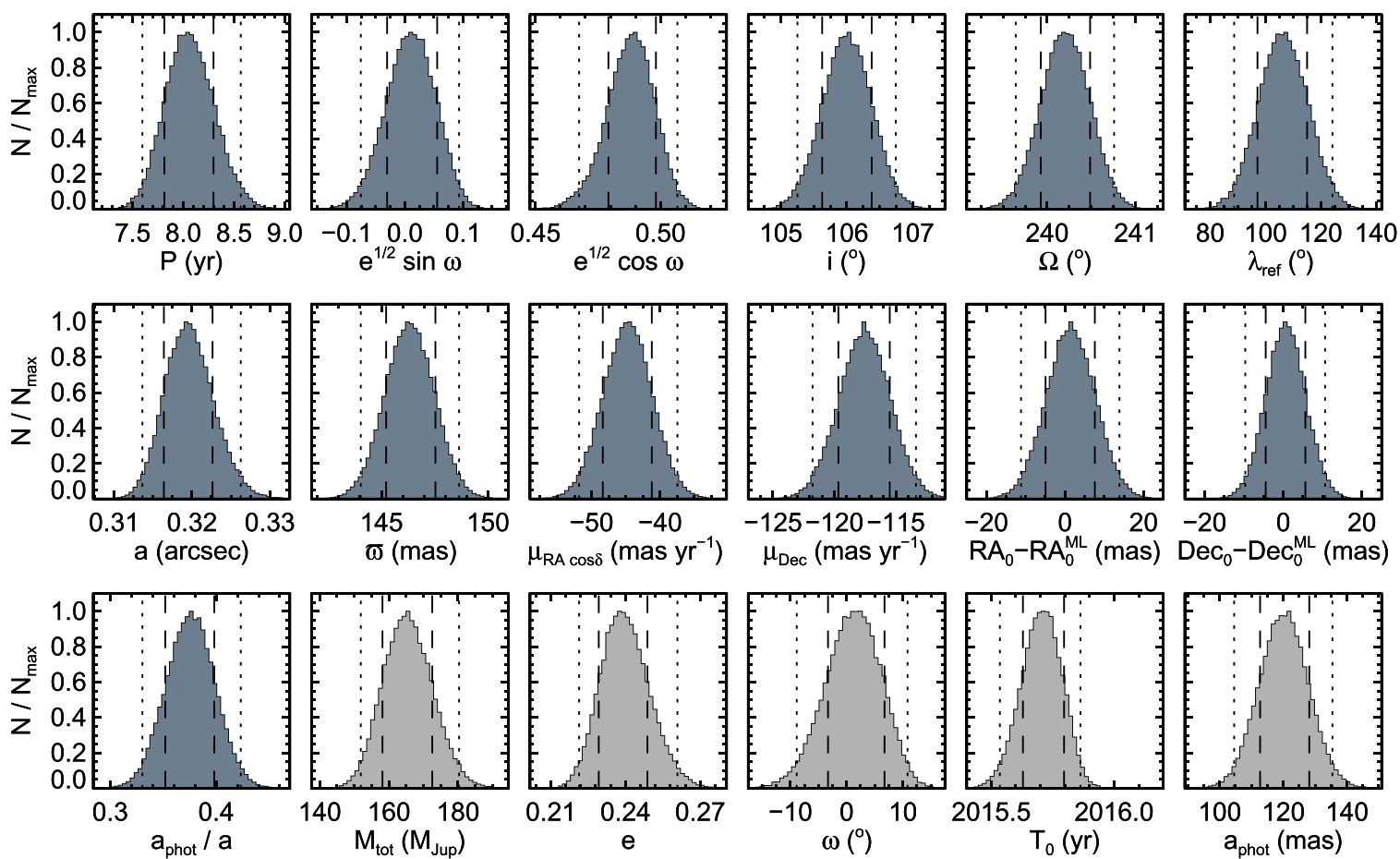

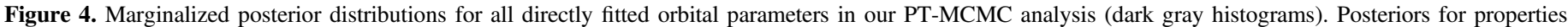
computed from the directly fitted parameters, like total mass and eccentricity, are shown in light gray histograms.

Table 3. Over just $\approx 4$ years of CFHT astrometric monitoring, nonlinear perturbations of up to 60 mas are observed. This enables a precise measurement of the photocenter orbit size $\left(120_{-7}^{+8}\right.$ mas). The ratio of the photocenter orbit size to the semimajor axis $\left(0.376_{-0.024}^{+0.022}\right)$ is related to the mass ratio, via the flux ratio in the CFHT bandpass. We used the $K$-band absolute magnitude of each component to compute their $K-K_{\mathrm{H} 2}$ color using the relations in Appendix A.2 of Dupuy \& Liu (2017), and thereby the flux ratio in the CFHT bandpass $K_{\mathrm{H} 2}$ of $0.0246 \pm 0.0016$. The very small amount of flux coming from the companion makes our mass ratio quite insensitive to the exact value of the flux ratio, such as that due to the variability of the primary star. The resulting mass ratio $\left(M_{2} / M_{1}=\right.$ $0.67_{-0.07}^{+0.06}$ ) yields individual masses of $99 \pm 6 M_{\text {Jup }}$ and $66 \pm 4 M_{\text {Jup }}$. Additional observational properties of the system are given in Table 4.

\subsection{Comparison with Previous Work}

Our absolute parallax $147.1_{-1.2}^{+1.1}$ mas is consistent with and considerably more precise than previously measured values of $142 \pm 38$ mas from Scholz (2014), which was based on catalog astrometry from Digital Sky Survey (DSS), SuperCOSMOS Sky Survey (SSS), Deep Near Infrared Survey (DENIS), Carlsberg Meridian Catalog (CMC) and WISE, and $165 \pm 30$ mas from Ivanov et al. (2015), which was based on combining their data with the Scholz (2014) astrometry. WISE J0720-0846 has photometry reported by Gaia but no proper motion or parallax solution in DR2, possibly due to its multiplicity or very red color.

Analysis of the orbit was performed by Burgasser et al. (2015b) based on their two epochs of NIRC2 imaging from 2014 and 2015 as well as RV monitoring from the Lick/Hamilton and Keck/NIRSPEC spectrographs. They concluded that the orbit was quite eccentric $\left(e=0.77_{-0.04}^{+0.02}\right)$ and nearly edge-on $\left(i=93^{\circ} .6_{-1.4}^{+1.6}\right)$ with a remarkably short period $\left(P=4.1_{-1.3}^{+2.7} \mathrm{yr}\right)$.
Our eccentricity ( $e=0.234_{-0.010}^{+0.009}$ ) is highly inconsistent with the analysis of Burgasser et al. (2015b), and our inclination and orbital period are larger by $7.5 \sigma$ and $1.4 \sigma$, respectively. The explanation for this disagreement is their 2014 January 19 UT epoch of astrometry, which was the original candidate detection. We did not use this data point in our orbit fit, and our MCMC posterior predicts a separation of $103 \pm 5$ mas and PA of $4^{\circ} \pm 8^{\circ}$ at that epoch. This differs from the astrometry of the candidate source identified by Burgasser et al. (2015a), which was at a separation of $139 \pm 14$ mas and PA of $262^{\circ} \pm 2^{\circ}$. Our predicted separation at that epoch is much tighter and was very likely not resolvable. For comparison, we have never resolved the binary at separations tighter than $\approx 190$ mas, and at one of our unresolved observation epochs (2016 October 11 UT) it is predicted to have been wider than the reported 2014 January separation, at $108.5 \pm 0.6$ mas. The FWHM of our imaging at that epoch was 51 mas, with a Strehl ratio of 0.10, while the data from Burgasser et al. (2015a) had an FWHM of 230 mas and Strehl ratio of 0.014. Therefore, we infer that the companion was not detectable in their imaging, and the candidate faint source was a PSF artifact. Visual inspection of the 2014 NIRC2 imaging obtained from the Keck Observatory Archive supports this conclusion.

Our orbit predicts the RV of the primary at the measurement epochs from Burgasser et al. (2015a) and Burgasser et al. (2015b) with a precision of $0.17-0.19 \mathrm{~km} \mathrm{~s}^{-1}$, which is typically 2-3 times smaller than their measurement errors. The positive slope of the RVs breaks the degeneracy between $\omega$ and $\omega+180^{\circ}$ in the astrometric orbit (i.e., at which node the companion is going into or coming out of the plane of the sky). Assuming the preferred value for $\omega$, then for the Lick/ Hamilton data the $\chi^{2}$ of the null hypothesis (constant RV) is 5.2 for 6 degrees of freedom (dof) and 4.2 after subtracting the orbital motion. For the reanalyzed NIRSPEC data reported by Burgasser et al. (2015b; not the originally published values from Burgasser et al. 2015a), the $\chi^{2}$ of the null hypothesis is 
Table 3

PT-MCMC Orbital Posteriors for WISE J0720-0846AB

\begin{tabular}{|c|c|c|c|}
\hline Property & Median $\pm 1 \sigma$ & $95.4 \%$ c.i. & Prior \\
\hline \multicolumn{4}{|c|}{ Fitted Parameters } \\
\hline Orbital period, $P(\mathrm{yr})$ & $8.06_{-0.25}^{+0.24}$ & $7.60,8.57$ & $1 / P$ (log-flat) \\
\hline$\sqrt{e} \sin \omega$ & $0.01 \pm 0.04$ & $-0.08,0.09$ & Uniform \\
\hline$\sqrt{e} \cos \omega$ & $0.488_{-0.009}^{+0.010}$ & $0.467,0.507$ & Uniform \\
\hline Inclination, $i\left(^{\circ}\right)$ & $106.0 \pm 0.4$ & $105.3,106.7$ & $\sin (i), 0^{\circ}<i<180^{\circ}$ \\
\hline R.A.ref - R.A.ref $(\mathrm{mas})$ & $1 \pm 6$ & $-11,14$ & Uniform \\
\hline decl.ref $_{\text {. }}-$ decl. $_{\text {ref }}^{\mathrm{ML}}$ (mas) & $1 \pm 5$ & $-10,11$ & Uniform \\
\hline Relative proper motion in R.A., $\mu_{\text {R.A.,rel }}\left({\left.\operatorname{mas~} \mathrm{yr}^{-1}\right)}^{-1}\right.$ & $-45_{-4}^{+3}$ & $-52,-37$ & Uniform \\
\hline Relative proper motion in decl., $\mu_{\text {decl.,rel }}\left(\operatorname{mas~yr}^{-1}\right)$ & $-117.5_{-2.1}^{+2.0}$ & $-121.8,-113.4$ & Uniform \\
\hline Relative parallax, $\varpi_{\text {rel }}$ (mas) & $146.3_{-1.1}^{+1.2}$ & $144.0,148.6$ & $1 / \varpi^{4}$ \\
\hline Ratio of photocenter orbit to semimajor axis, $a_{\text {phot }} / a$ & $0.376_{-0.024}^{+0.022}$ & $0.331,0.423$ & Uniform \\
\hline Argument of periastron, $\omega\left(^{\circ}\right)$ & $1 \pm 5$ & $-9,11$ & $\ldots$ \\
\hline Time of periastron, $T_{0}=t_{\text {ref }}-P \frac{\lambda-\omega}{360^{\circ}}(\mathrm{JD})$ & $2457282_{-28}^{+33}$ & 2457219,2457339 & $\cdots$ \\
\hline Photocenter semimajor axis, $a_{\mathrm{phot}}$ (mas) & $120_{-7}^{+8}$ & 105,135 & $\ldots$ \\
\hline$\left(a^{3} P^{-2}\right) \times 10^{4}\left(\operatorname{arcsec}^{3} \mathrm{yr}^{-2}\right)$ & $5.02 \pm 0.19$ & $4.66,5.41$ & $\ldots$ \\
\hline Correction to absolute R.A. proper motion, $\Delta \mu_{\text {R.A. }}\left(\right.$ mas $\left.\mathrm{yr}^{-1}\right)$ & $-1.42_{-0.12}^{+0.16}$ & $-1.67,-1.06$ & $\cdots$ \\
\hline Correction to absolute decl. proper motion, $\Delta \mu_{\text {decl. }}\left(\right.$ mas $\left.\mathrm{yr}^{-1}\right)$ & $0.97_{-0.19}^{+0.20}$ & $0.58,1.37$ & $\ldots$ \\
\hline Correction to absolute parallax, $\Delta \varpi$ (mas) & $0.77_{-0.05}^{+0.04}$ & $0.68,0.88$ & $\ldots$ \\
\hline Absolute proper motion in R.A., $\mu_{\text {R.A. }}\left({\left.\text { mas } \mathrm{yr}^{-1}\right)}^{-1}\right.$ & $-46_{-3}^{+4}$ & $-53,-39$ & .. \\
\hline Absolute proper motion in decl., $\mu_{\text {decl. }}\left(\right.$ mas yr $\left.^{-1}\right)$ & $-116.5_{-2.0}^{+2.2}$ & $-120.8,-112.3$ & $\ldots$ \\
\hline Absolute parallax, $\varpi$ (mas) & $147.1_{-1.2}^{+1.1}$ & $144.8,149.4$ & $\ldots$ \\
\hline Distance, $d(\mathrm{pc})$ & $6.80_{-0.06}^{+0.05}$ & $6.69,6.90$ & $\ldots$ \\
\hline
\end{tabular}

Note. The full thirteen-parameter fit has $\chi^{2}=25.8$ (21 dof), and the relative orbit has $\chi^{2}=4.84$ (7 dof). The orbit quality metrics defined by Dupuy \& Liu (2017) are $\delta \log M_{\mathrm{tot}}=0.033 \mathrm{dex}, \delta e=0.020$, and $\Delta t_{\mathrm{obs}} / P=0.47$, indicating a high-quality orbit determination. Maximum-likelihood coordinates at the reference epoch (2010.0): (R.A ., decl. $)_{\mathrm{ref}}^{\mathrm{ML}}=(110.0135245,-08.7809247)$.

35.9 (4 dof) and 10.1 after subtracting the orbital motion. This indicates that the general RV trend was detected in these data, although the RV measurement errors are likely somewhat underestimated. After subtracting the orbital motion, we compute system velocities from the two RV data sets of $82.2 \pm 0.4 \mathrm{~km} \mathrm{~s}^{-1}$ and $83.1 \pm 0.7 \mathrm{~km} \mathrm{~s}^{-1}$, from which we compute a weighted average of $82.4 \pm 0.3 \mathrm{~km} \mathrm{~s}^{-1}$. This is slightly smaller than the RV of $83.1 \pm 0.4 \mathrm{~km} \mathrm{~s}^{-1}$ derived by Burgasser et al. (2015a) because most of their measurements were obtained when the RV of the primary was $>0 \mathrm{~km} \mathrm{~s}^{-1}$. Figure 5 displays our predicted RV orbit for the primary alongside the measurements.

\section{Spectroscopic Analysis}

We determined the bolometric flux $\left(f_{\mathrm{bol}}\right)$ of the combined light of the WISE J0720-0846 system following the same procedure as Mann et al. (2015), which we briefly summarize here. We simultaneously combined and absolutely calibrated the SNIFS and SpeX spectra to photometry from Gaia DR2 (Evans et al. 2018), the 2MASS (Skrutskie et al. 2006), and the WISE (Wright et al. 2010), using the appropriate filter profiles and zero points (Cohen et al. 2003; Maíz Apellániz \& Weiler 2018) to generate synthetic photometry from the spectrum. We assumed zero reddening, as the target is at $\approx 7 \mathrm{pc}$. To account for higher stellar variability in the optical, we adopted photometric errors of $0.05 \mathrm{mag}$ in the $B p$ band and 0.04 mag in the $G$ and $R p$ bands. To create a full spectral energy distribution (SED), we filled in gaps in the spectral coverage (e.g., beyond $2.4 \mu \mathrm{m}$ ) using the best-fit BT-Settl models (Allard et al. 2013).

Figure 6 shows the observed and model spectra we used, as well as the photometric residuals. To calculate $f_{\text {bol }}$ from the SED, we integrated the combined and calibrated spectrum over all wavelengths. Errors on $f_{\text {bol }}$ account for measurement errors in the observed spectrum, the range of possible BT-Settl models that can fit the data, as well as errors in filter profiles and zero points. This analysis yields an $f_{\text {bol }}$ of $(2.28 \pm 0.07) \times 10^{-10} \mathrm{erg} \mathrm{cm}^{-2} \mathrm{~s}^{-1}$ for the combined light of the system.

Using a single-component model, our analysis implicitly assumes that the contribution of the T-dwarf companion to the combined-light SED is negligible relative to photometric and BT-Settl model uncertainties. This is certainly the case in the optical, and conversely it is unlikely to be true at long wavelengths, which is why we did not include $W 3$ and $W 4$ in our analysis. 
Table 4

Observational Properties of WISE J0720-0846AB

\begin{tabular}{|c|c|c|}
\hline Property & Value & References \\
\hline \multicolumn{3}{|c|}{ Integrated Light } \\
\hline SpT (opt) & M9.5 & $\mathrm{B} 15 \mathrm{~b}$ \\
\hline SpT (NIR) & M9.8 FLD-G & G15 \\
\hline$Y(\mathrm{mag})$ & $11.56 \pm 0.06$ & ${ }^{*}, \mathrm{~B} 19$ \\
\hline$J(\mathrm{mag})$ & $10.587 \pm 0.023$ & *, B19 \\
\hline$H$ (mag) & $9.982 \pm 0.019$ & $*$, B19 \\
\hline $\mathrm{CH}_{4 s}$ (mag) & $9.999 \pm 0.019$ & ${ }^{*}, \mathrm{~B} 19$ \\
\hline$K(\mathrm{mag})$ & $9.446 \pm 0.019$ & *, B19 \\
\hline$d(\mathrm{pc})$ & $6.80_{-0.06}^{+0.05}$ & * \\
\hline$M\left(M_{\text {Jup }}\right)$ & $165 \pm 7$ & * \\
\hline$f_{\mathrm{bol}}\left(\mathrm{erg} \mathrm{cm}^{-2} \mathrm{~s}^{-1}\right)$ & $(2.28 \pm 0.07) \times 10^{-10}$ & * \\
\hline$L_{\text {bol }}\left(L_{\odot}\right)$ & $(3.44 \pm 0.13) \times 10^{-4}$ & * \\
\hline \multicolumn{3}{|c|}{ WISE J0720-0846A } \\
\hline SpT (NIR) & M9.5 \pm 0.5 & B15a \\
\hline$Y(\mathrm{mag})$ & $11.63 \pm 0.06$ & ${ }^{*}, \mathrm{~B} 19$ \\
\hline$J(\mathrm{mag})$ & $10.684 \pm 0.023$ & *, B19 \\
\hline$H$ (mag) & $10.037 \pm 0.019$ & *, B19 \\
\hline $\mathrm{CH}_{4 s}$ (mag) & $10.066 \pm 0.019$ & ${ }^{*}, \mathrm{~B} 19$ \\
\hline$K(\mathrm{mag})$ & $9.479 \pm 0.019$ & ${ }^{*}, \mathrm{~B} 19$ \\
\hline$M\left(M_{\mathrm{Jup}}\right)$ & $99 \pm 6$ & * \\
\hline$f_{\text {bol }}\left(\mathrm{erg} \mathrm{cm}^{-2} \mathrm{~s}^{-1}\right)$ & $(2.18 \pm 0.07) \times 10^{-10}$ & * \\
\hline$L_{\text {bol }}\left(L_{\odot}\right)$ & $(3.29 \pm 0.13) \times 10^{-4}$ & * \\
\hline \multicolumn{3}{|c|}{ WISE J0720-0846B } \\
\hline SpT (IR) & $\mathrm{T} 5.5 \pm 0.5$ & B15a \\
\hline$Y$ (mag) & $14.54 \pm 0.07$ & *, B19 \\
\hline$J(\mathrm{mag})$ & $13.26 \pm 0.04$ & ${ }^{*}, \mathrm{~B} 19$ \\
\hline$H$ (mag) & $13.24 \pm 0.04$ & * B19 \\
\hline $\mathrm{CH}_{4 s}$ (mag) & $13.05 \pm 0.04$ & *, B19 \\
\hline$K(\mathrm{mag})$ & $13.31 \pm 0.07$ & *, B19 \\
\hline$M\left(M_{\mathrm{Jup}}\right)$ & $66 \pm 4$ & * \\
\hline$f_{\text {bol }}\left(\mathrm{erg} \mathrm{cm}^{-2} \mathrm{~s}^{-1}\right)$ & $(1.02 \pm 0.17) \times 10^{-11}$ & * \\
\hline$L_{\mathrm{bol}}\left(L_{\odot}\right)$ & $(1.5 \pm 0.3) \times 10^{-5}$ & * \\
\hline
\end{tabular}

Note. All photometry on the MKO system.

References. ( ${ }^{*}$ ) this work; (B15a) Burgasser et al. (2015b); (B15b) Burgasser et al. (2015a); (B19) W. M. J. Best et al. (2019, in preparation); (G15) Gagné et al. (2015).

In the NIR, we must consider the binary separation and the fact that we used a 0!" 3 slit for our observations. Using our orbit determination, we predict the separation and PA of the binary at this epoch was $348.7 \pm 1.6$ mas and $67^{\circ} .69 \pm 0.11$. This is nearly orthogonal to the parallactic angle-aligned slit used for our observations $\left(100^{\circ} .20\right.$ different). Assuming that the slit was centered on the primary, and a Gaussian PSF with an FWHM of 0.19 , the companion contribution to our spectrum was $32 \%$ smaller than if the slit were aligned with the binary PA. Thus, the companion contribution is still quite significant, so we performed tests fitting two-component SEDs to our data, one for each binary component instead of just one for the primary, and calculated the impact on our final derived $f_{\text {bol }}$. The BT-Settl models we used for these tests do not reproduce the companion photometry well, possibly due to deficiencies in the atmospheric physics, and the model grid is only coarsely sampled in $T_{\text {eff }}$, making accurate SED fitting unfeasible. Therefore, while the two-component fits are not suitable for accurate SED modeling, they do provide an estimate of the

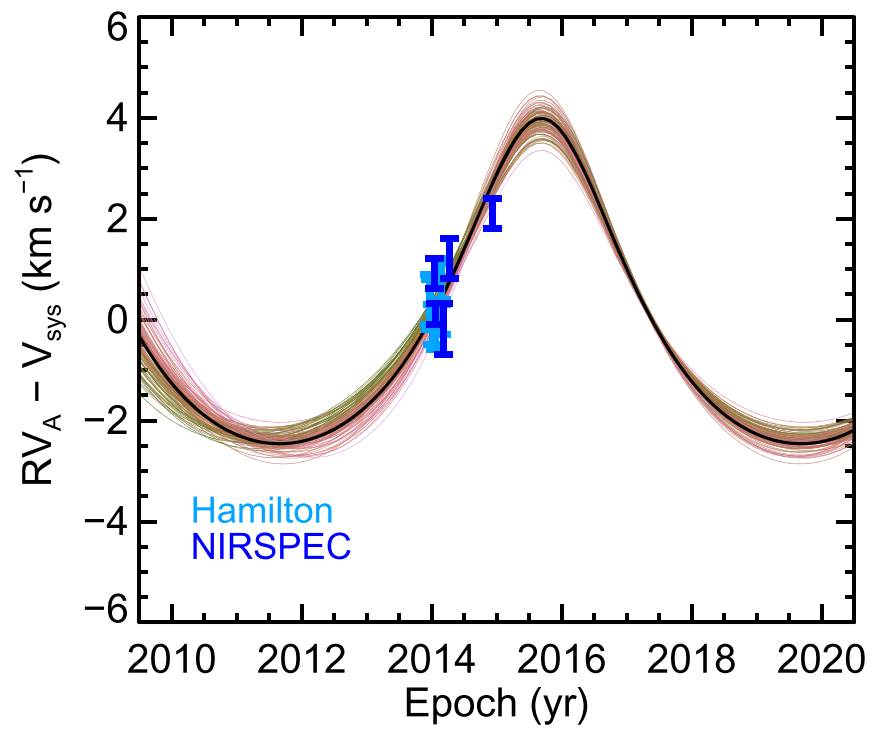

Figure 5. RV orbit of the late-M primary, predicted from our astrometric analysis, plotted over more than one full period alongside measurements from Burgasser et al. (2015b, 2015a). The mean system velocity has been subtracted off of each set of measurements. The best-fit orbit is a thick black line, and 100 orbit solutions drawn randomly from our MCMC posterior are thin lines colored according to the dynamical total mass (same as Figure 3).

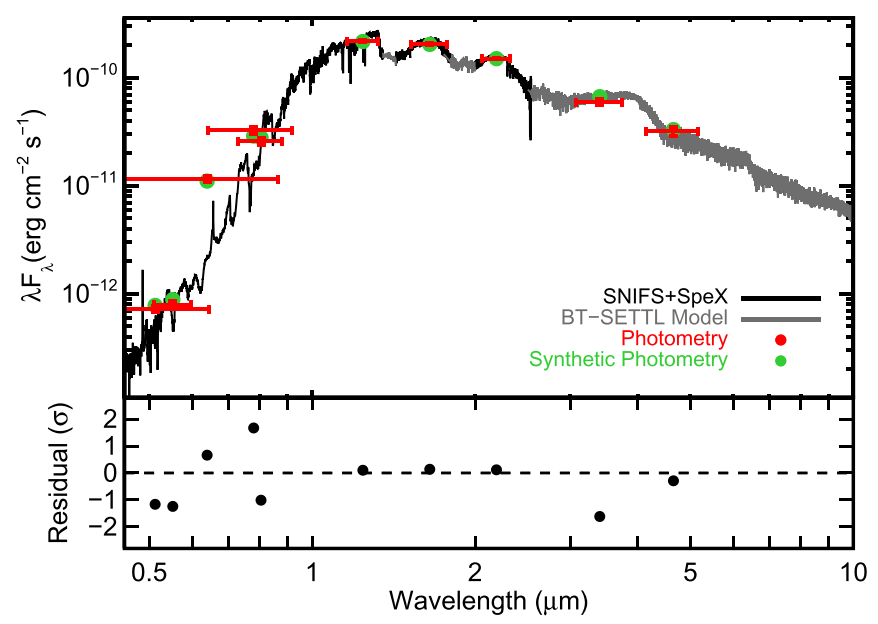

Figure 6. Top: absolutely calibrated spectrum from SNIFS and SpeX (black), best-fit BT-Settl model (gray), as well as literature (red) and synthetic (green) photometry for WISE J0720-0846 in combined light. Horizontal error bars indicate the width of the filter, while vertical error bars are the measurement errors. Both the observed spectrum and photometry are expected to contain flux from the faint companion, and by fitting a model appropriate for the primary only we implicitly assume what our measured flux ratios indicate, that the companion flux is negligible in the optical and NIR. Bottom: residuals between observed and synthetic photometry in standard deviations.

error introduced by assuming a single-component model for the SED fitting. We find that including a wide range of models still resulted in at most $\pm 1 \%$ of variation in the derived $f_{\mathrm{bol}}$. This is negligible compared to our $f_{\text {bol }}$ measurement error of $3.1 \%$.

Our combined-light $f_{\mathrm{bol}}$ and measured parallax yield a total bolometric luminosity of $(3.44 \pm 0.13) \times 10^{-4} L_{\odot}$. We computed the bolometric luminosity of the T-dwarf companion from the $K$-band absolute magnitude relation of Dupuy \& Liu (2017), finding $L_{\mathrm{bol}}=(1.5 \pm 0.3) \times 10^{-5} L_{\odot}$, which is $4.4 \%$ of the total bolometric flux. Converting this back to $f_{\text {bol }}=(1.02 \pm 0.17) \times$ $10^{-11} \mathrm{erg} \mathrm{cm}^{-2} \mathrm{~s}^{-1}$, and subtracting it from the combined-light 
Table 5

Fundamental Properties of WISE J0720-0846AB

\begin{tabular}{|c|c|c|c|c|c|c|}
\hline \multirow[b]{2}{*}{ Property } & \multicolumn{3}{|c|}{ Using Total Mass } & \multicolumn{3}{|c|}{ Using Individual Masses } \\
\hline & Primary & Secondary & $\Delta=B-A$ & Primary & Secondary & $\Delta=B-A$ \\
\hline \multicolumn{7}{|c|}{ Input Observed Properties } \\
\hline Mass ratio $q$ & $\ldots$ & $\ldots$ & $\cdots$ & \multicolumn{2}{|c|}{$0.67_{-0.07}^{+0.06}$} & $\cdots$ \\
\hline $\log \left(L_{\mathrm{bol}}\right)\left(L_{\odot}\right)$ & $-3.503 \pm 0.016$ & $-4.82 \pm 0.07$ & $-1.32 \pm 0.08$ & $\ldots$ & $\cdots$ & $\cdots$ \\
\hline
\end{tabular}

Derived from BHAC15 Evolutionary Models

\begin{tabular}{|c|c|c|c|c|c|c|}
\hline Mass $M\left(M_{\text {Jup }}\right)$ & $\cdots$ & $\cdots$ & $\cdots$ & $86.0_{-0.5}^{+0.6}$ & $\cdots$ & $\cdots$ \\
\hline $\log \left(L_{\mathrm{bol}}\right)\left(L_{\odot}\right)$ & $\cdots$ & $\cdots$ & $\cdots$ & $-3.505_{-0.017}^{+0.016}$ & $\cdots$ & $\cdots$ \\
\hline Age $t$ (Gyr) & & & $\ldots$ & $5.5_{-3.2}^{+2.9}$ & $\cdots$ & $\ldots$ \\
\hline $\log (t)(\mathrm{yr})$ & & & $\ldots$ & $9.74_{-0.15}^{+0.26}$ & $\ldots$ & $\ldots$ \\
\hline Radius ( $R_{\text {Jup }}$ ) & $\cdots$ & $\cdots$ & $\cdots$ & $0.992_{-0.007}^{+0.006}$ & $\cdots$ & $\ldots$ \\
\hline $\log (g)\left(\mathrm{cm} \mathrm{s}^{-2}\right)$ & $\cdots$ & $\cdots$ & $\cdots$ & $5.3363_{-0.0029}^{+0.0036}$ & $\cdots$ & $\ldots$ \\
\hline $\log \left(\mathrm{Li} / \mathrm{Li}_{\text {init }}\right)$ & $\cdots$ & $\cdots$ & $\cdots$ & $<-4.0$ & $\cdots$ & $\cdots$ \\
\hline $\operatorname{MKO}(J-H)(\mathrm{mag})$ & $\cdots$ & $\cdots$ & $\cdots$ & $0.448 \pm 0.005$ & $\cdots$ & $\cdots$ \\
\hline
\end{tabular}

\begin{tabular}{|c|c|c|c|c|c|c|}
\hline \multicolumn{7}{|c|}{ Derived from SM08 Hybrid Evolutionary Models } \\
\hline Mass $M\left(M_{\text {Jup }}\right)$ & $\cdots$ & $\cdots$ & $\cdots$ & $\cdots$ & $66.5_{-2.0}^{+3.5}$ & $\cdots$ \\
\hline $\log \left(L_{\mathrm{bol}}\right)\left(L_{\odot}\right)$ & $\cdots$ & $\cdots$ & $\cdots$ & $\cdots$ & $-4.81 \pm 0.07$ & $\cdots$ \\
\hline Age $t(\mathrm{Gyr})$ & \multicolumn{2}{|c|}{$\cdots$} & $\cdots$ & $\cdots$ & $6.8_{-3.1}^{+2.2}$ & $\cdots$ \\
\hline $\log (t)(y r)$ & \multicolumn{2}{|c|}{$\cdots$} & $\ldots$ & $\cdots$ & $9.83_{-0.19}^{+0.17}$ & $\ldots$ \\
\hline$T_{\text {eff }}(\mathrm{K})$ & $\cdots$ & $\cdots$ & $\cdots$ & $\cdots$ & $1250 \pm 40$ & $\cdots$ \\
\hline Radius $\left(R_{\mathrm{Jup}}\right)$ & $\ldots$ & $\ldots$ & $\ldots$ & $\ldots$ & $0.822_{-0.016}^{+0.015}$ & $\cdots$ \\
\hline $\log (g)\left(\mathrm{cm} \mathrm{s}^{-2}\right)$ & $\ldots$ & $\ldots$ & $\ldots$ & $\ldots$ & $5.387_{-0.023}^{+0.033}$ & $\ldots$ \\
\hline $\operatorname{MKO}(J-K)(\mathrm{mag})$ & $\ldots$ & $\ldots$ & $\ldots$ & $\ldots$ & $0.802_{-0.406}^{+0.366}$ & $\ldots$ \\
\hline $\operatorname{MKO}(J-H)(\operatorname{mag})$ & $\cdots$ & $\cdots$ & $\cdots$ & $\cdots$ & $0.638_{-0.180}^{+0.176}$ & $\cdots$ \\
\hline \multicolumn{7}{|c|}{ Derived from Cond Evolutionary Models } \\
\hline Mass $M\left(M_{\text {Jup }}\right)$ & $86.7 \pm 0.5$ & $70.7_{-1.7}^{+2.4}$ & $-16.1_{-1.8}^{+2.6}$ & $86.7_{-0.5}^{+0.6}$ & $67.6_{-2.9}^{+4.2}$ & $-19_{-3}^{+4}$ \\
\hline $\log \left(L_{\mathrm{bol}}\right)\left(L_{\odot}\right)$ & $-3.506_{-0.016}^{+0.017}$ & $-4.82 \pm 0.07$ & $-1.31 \pm 0.07$ & $-3.506_{-0.017}^{+0.015}$ & $-4.83 \pm 0.07$ & $-1.32 \pm 0.07$ \\
\hline Mass ratio $q$ & \multicolumn{2}{|c|}{$0.815_{-0.020}^{+0.029}$} & $\ldots$ & \multicolumn{2}{|c|}{$0.78_{-0.03}^{+0.05}$} & $\ldots$ \\
\hline Age $t$ (Gyr) & \multicolumn{2}{|c|}{$7.4_{-1.1}^{+2.5}$} & $\ldots$ & $5.4_{-3.5}^{+2.6}$ & $5.3_{-2.5}^{+1.5}$ & $0 \pm 3$ \\
\hline $\log (t)(\mathrm{yr})$ & \multicolumn{2}{|c|}{$9.87_{-0.06}^{+0.13}$} & $\ldots$ & $9.74_{-0.15}^{+0.26}$ & $9.73_{-0.15}^{+0.19}$ & $0.01_{-0.32}^{+0.27}$ \\
\hline$T_{\text {eff }}(\mathrm{K})$ & $2404 \pm 15$ & $1290 \pm 50$ & $-1110 \pm 50$ & $2404_{-15}^{+14}$ & $1270 \pm 50$ & $-1130 \pm 50$ \\
\hline Radius ( $\left.R_{\text {Jup }}\right)$ & $1.000 \pm 0.006$ & $0.767_{-0.008}^{+0.013}$ & $-0.232_{-0.012}^{+0.011}$ & $1.000 \pm 0.006$ & $0.776_{-0.014}^{+0.013}$ & $-0.223_{-0.017}^{+0.014}$ \\
\hline $\log (g)\left(\mathrm{cm} \mathrm{s}^{-2}\right)$ & $5.333 \pm 0.003$ & $5.473_{-0.010}^{+0.023}$ & $0.140_{-0.013}^{+0.024}$ & $5.3323_{-0.0026}^{+0.0038}$ & $5.447_{-0.021}^{+0.049}$ & $0.115_{-0.027}^{+0.045}$ \\
\hline $\log \left(\mathrm{Li} / \mathrm{Li}_{\text {init }}\right)$ & $\ldots$ & $\ldots$ & $\ldots$ & $\ldots$ & $-0.0210 \pm 0.0013$ & $\ldots$ \\
\hline $\operatorname{MKO}(J-K)(\mathrm{mag})$ & $0.649 \pm 0.005$ & $-0.166_{-0.044}^{+0.052}$ & $-0.815_{-0.045}^{+0.052}$ & $0.650_{-0.006}^{+0.004}$ & $-0.167_{-0.053}^{+0.056}$ & $-0.816_{-0.053}^{+0.057}$ \\
\hline $\operatorname{MKO}(J-H)(\mathrm{mag})$ & $0.316 \pm 0.004$ & $-0.283_{-0.023}^{+0.019}$ & $-0.599_{-0.022}^{+0.021}$ & $0.317 \pm 0.004$ & $-0.287_{-0.024}^{+0.0023}$ & $-0.603 \pm 0.024$ \\
\hline
\end{tabular}

Note. The BHAC15 models do not extend to the luminosity of the secondary, so for the BHAC individual-mass analysis only the results for WISE J0720-0846A are given. The SM08 models do not extend to the luminosity of the primary, so for the SM08 individual-mass analysis only the results for WISE J0720-0846B are given.

flux, gives $f_{\text {bol }}=(2.18 \pm 0.07) \times 10^{-10} \mathrm{erg} \mathrm{cm}^{-2} \mathrm{~s}^{-1}$ and $L_{\text {bol }}=(3.29 \pm 0.13) \times 10^{-4} L_{\odot}$ for the primary component.

From our SpeX spectrum, we also derived a metallicity of $[\mathrm{Fe} / \mathrm{H}]=+0.15 \pm 0.10 \mathrm{dex}$ using the calibration of Mann et al. (2014). It is based on the correlation between metallicity and the strength of atomic $\mathrm{Na}, \mathrm{Ca}$, and $\mathrm{K}$ lines in NIR spectra of $\mathrm{M}$ dwarfs, validated using wide binaries containing an FGK primary and a late-M companion (e.g., Bonfils et al. 2005; Rojas-Ayala et al. 2012). Given the lack of these same alkali features in mid-T-dwarf spectra, and the faintness of the T-dwarf companion here, the influence of the companion on our metallicity analysis is expected to be negligible.

\section{Evolutionary Model Analysis}

In order to derive additional fundamental parameters for the system components, we have combined our measured masses and luminosities with evolutionary models. We perform a rejection sampling analysis that is the same as we used in Dupuy \& Liu (2017). Briefly, we start with randomly drawn masses and luminosities from our measured distributions, tracking the covariance in these parameters, and combine these with randomly drawn ages according to a uniform prior in time. Each random draw corresponds to a measured luminosity as well as a model-derived luminosity (from mass and age), and the rejection probability is computed from the difference 

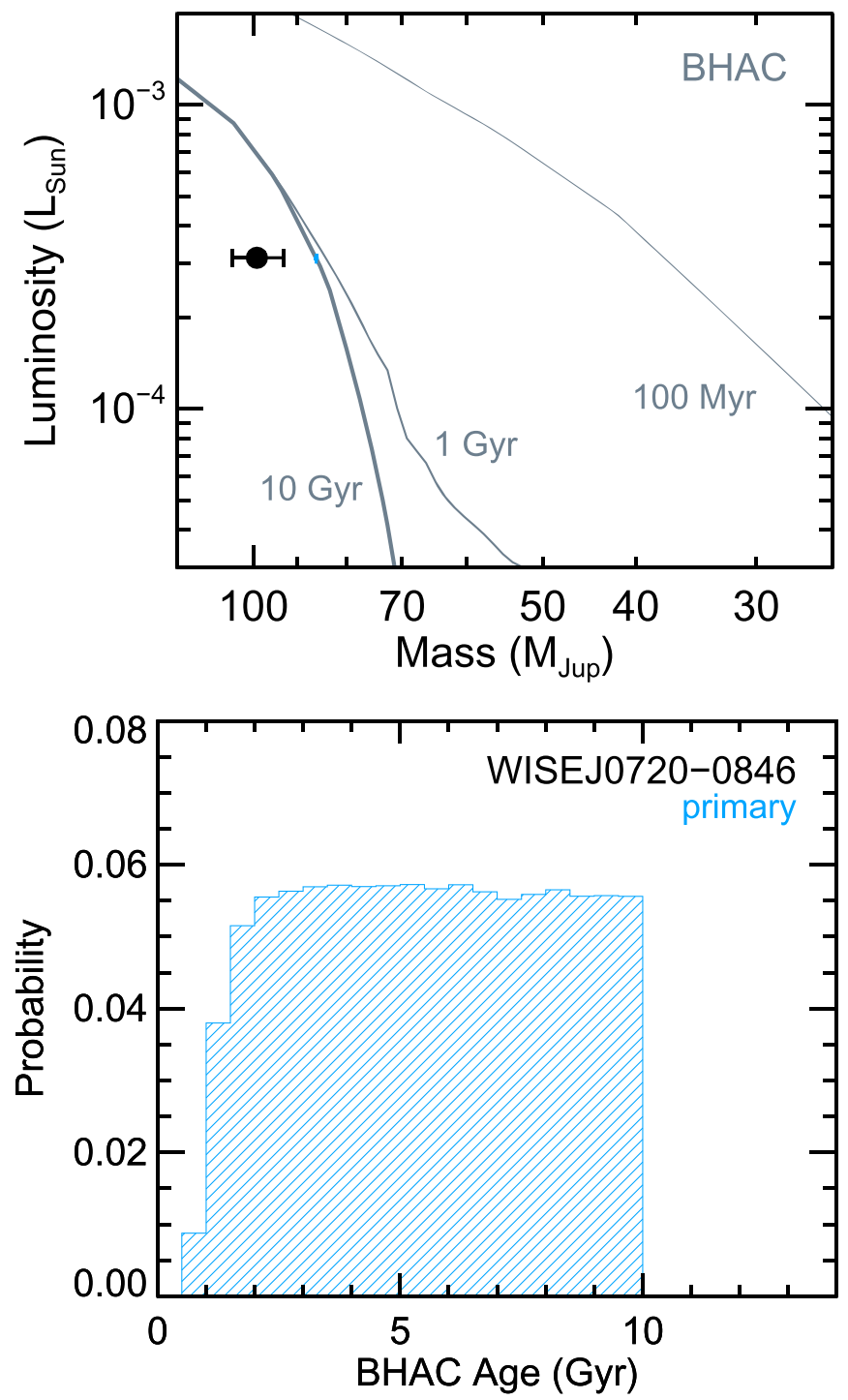

Figure 7. Top: our measured mass and luminosity for WISE J0720-0846A shown alongside the BHAC15 isochrones that are appropriate for a late-M dwarf. WISE J0720-0846A appears massive for its luminosity and is marginally inconsistent with models $(2.1 \sigma)$. Bottom: age distribution from our individual-mass analysis of WISE J0720-0846A. Given that we determine WISE J0720-0846A to be a star, its age is consistent with the full range of main-sequence ages covered by the BHAC15 models ( $<10 \mathrm{Gyr})$, and the shape of the distribution is simply the result of our uniform age prior.

between these two luminosities. Over three iterations we adjust the range over which ages are drawn as needed to ensure a well-sampled posterior on model-derived properties. In addition to this individual-mass analysis, we also perform a totalmass analysis where only the resolved luminosities and dynamical total mass are input as measurements. In this case random component masses and a system age are drawn for each trial, and the rejection probability is computed from the component luminosities and summed mass.

Table 5 shows the results of our evolutionary model analysis, which we performed for three different sets of models. The (Baraffe et al. 2015, hereinafter BHAC15) grid encompasses the luminosity of WISE J0720-0846A but not its much cooler companion, so we only report the individual-mass analysis for the primary with these models. Conversely, the Saumon \& Marley (2008, hereinafter SM08) models cover the cooler WISE J0720-0846B but not the primary. We use the hybrid
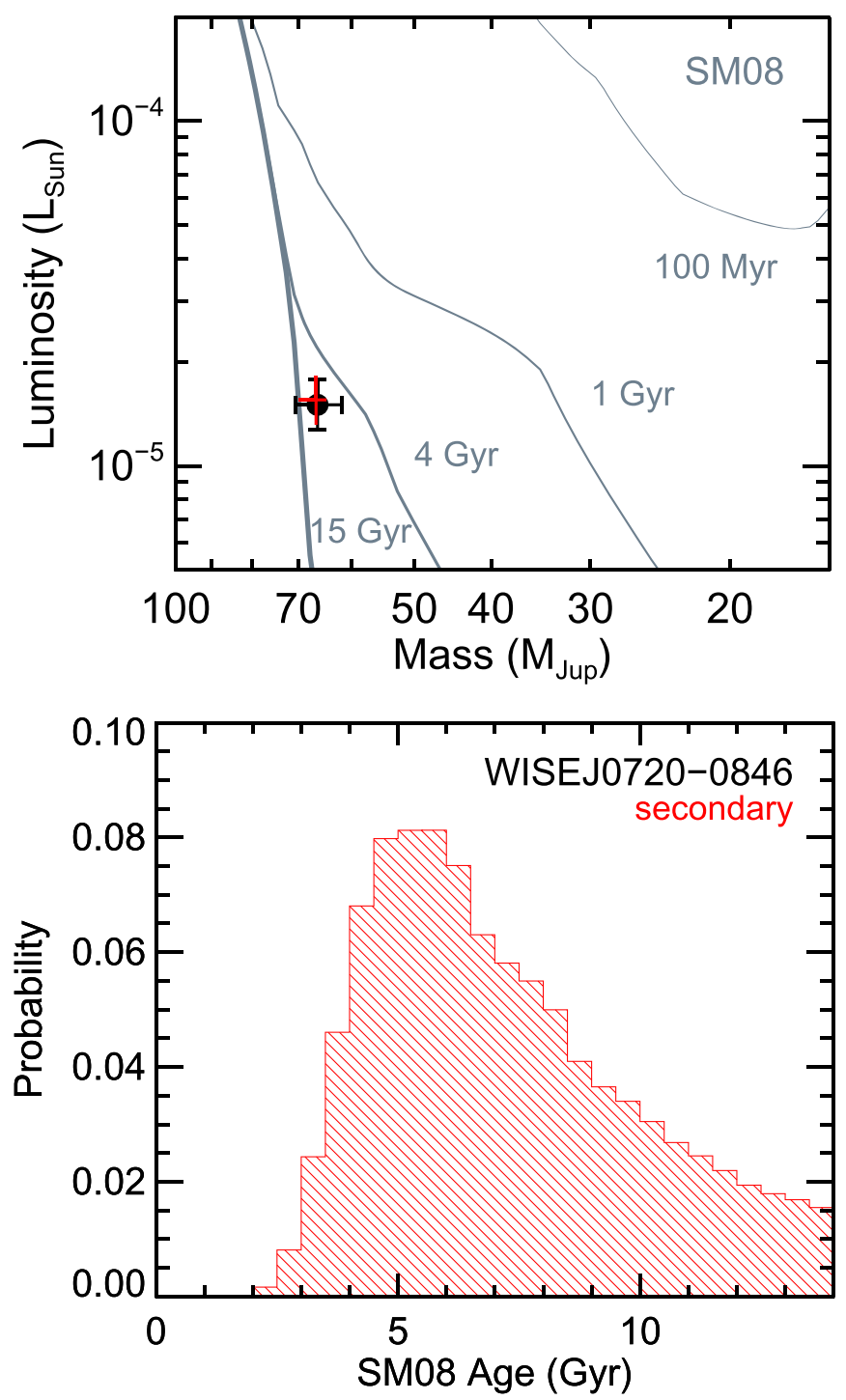

Figure 8. Top: our measured mass and luminosity for WISE J0720-0846B shown alongside the SM08 hybrid isochrones, which are appropriate for a mid$\mathrm{T}$ dwarf. WISE J0720-0846B is rather massive for its luminosity but consistent with models at old ages. The colored symbol indicates the $1 \sigma$ posterior on mass and luminosity after our rejection sampling analysis. Bottom: age distribution from our individual-mass analysis of WISE J0720-0846B, which is based on our input mass and luminosity measurements and a uniform prior on age. Given how broad the distribution is, it is strongly influenced by our prior.

version of the SM08 models, that transition from cloudy to cloud-free atmospheres as objects cool from $1400 \mathrm{~K}$ to $1200 \mathrm{~K}$, as they are more appropriate for an object like WISE J0720 $-0846 \mathrm{~B}$ that is on the blue end of the $\mathrm{L} / \mathrm{T}$ transition and may still possess clouds. Finally, we include the Cond models (Baraffe et al. 2003) because they are the only models to cover the physical properties of both components. However, unlike the above models, Cond does not include condensate clouds in the photosphere and thus should not be physically appropriate for either component.

WISE J0720-0846A has a high mass for its luminosity, making it marginally inconsistent with models $(2.1 \sigma$, Figure 7$)$. Future refinement of the total mass and, more importantly, mass ratio could resolve this discrepancy. For example, our total-mass analysis that does not use the mass ratio gives a selfconsistent result with a slightly lower mass for the primary and 


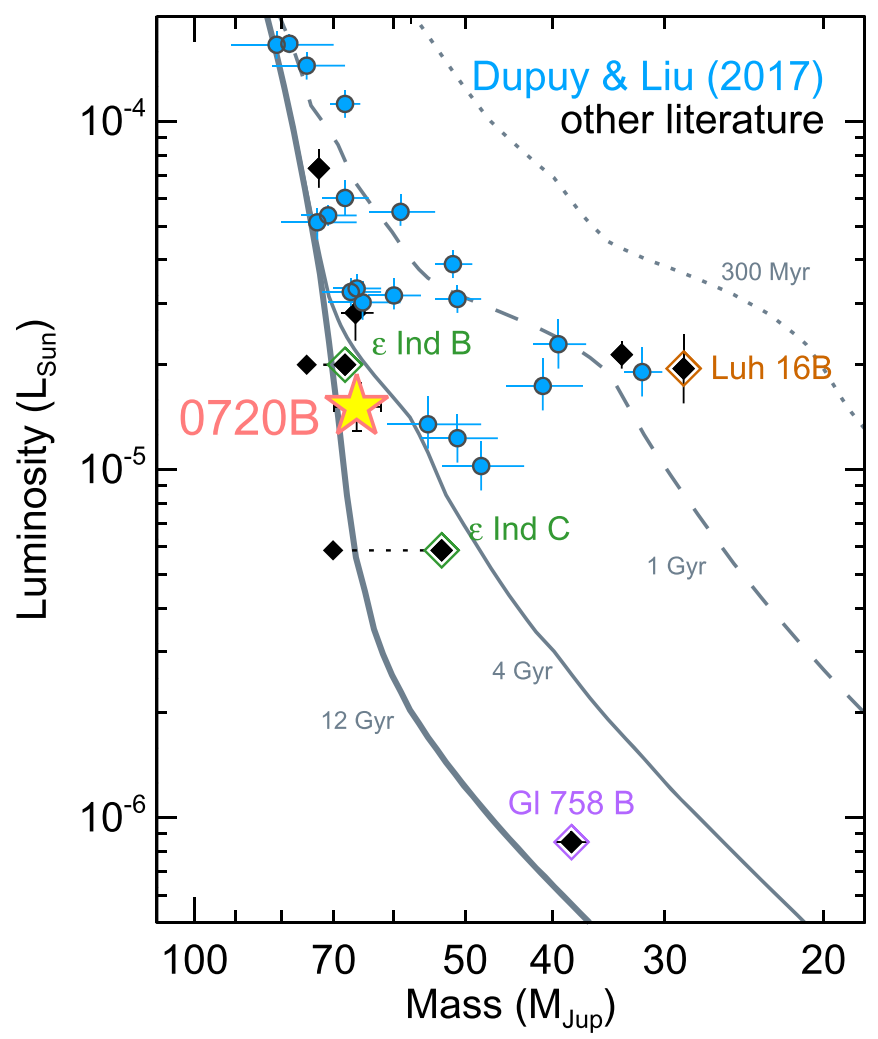

Figure 9. Luminosity as a function of mass for ultracool dwarfs that have model-independent mass measurements, with gray lines showing SM08 hybrid evolutionary model isochrones. Most measurements come from Dupuy \& Liu (2017, blue circles), and other literature measurements are plotted as black diamonds. Notable literature $\mathrm{T}$ dwarfs are highlighted with colored diamonds: $\epsilon$ Ind B and C (green), ${ }^{* *}$ LUH 16B (brown), and Gl 758B (purple). For $\epsilon$ Ind BC, we plot both the lower masses from Cardoso (2012) and higher masses from Dieterich et al. (2018), connected by horizontal dotted lines. Like $\epsilon$ Ind B, our mass measurement for WISE J0720-0846B is higher than other objects of comparable luminosity.

higher mass for the secondary, making the mass ratio 0.81 , which is exactly at the edge of our $95.4 \%$ credible interval of $0.55-0.81$.

Given that the mass and luminosity of WISE J0720-0846A are above the theoretical substellar boundary, our model analysis yields no age information from it. WISE J0720 $-0846 \mathrm{~B}$ on the other hand is only consistent with relatively old ages ( $\gtrsim 4$ Gyr), although even this quantitative age limit is influenced by our prior assumption of uniform age given the broad output posterior (Figure 8).

Table 5 gives physical properties derived from our evolutionary model analysis, including our posteriors on the masses and luminosities. Every Monte Carlo trial preserved in our rejection sampling analysis corresponds to a part of parameter space actually covered by models, so our posterior primary mass of $86.0_{-0.5}^{+0.6} M_{\text {Jup }}$ (BHAC15) is significantly smaller, with smaller errors, than our input measurement. The primary's model-derived effective temperature $\left(T_{\text {eff }}\right)$ of $2407_{-15}^{+14} \mathrm{~K}$ is consistent with its spectral type of M9.5, as the mass-calibrated spectral type- $T_{\text {eff }}$ relation of Dupuy \& Liu (2017) based on the same models gives $2400 \pm 90 \mathrm{~K}$. Likewise, the SM08-based relation gives $T_{\text {eff }}=1180 \pm 80 \mathrm{~K}$ for WISE J0720-0846B, which agrees with our mass-calibrated value of $1250 \pm 40 \mathrm{~K}$. The smaller radius of the secondary $\left(0.822_{-0.016}^{+0.015} R_{\text {Jup }}\right)$ relative to the primary $\left(0.992_{-0.007}^{+0.006} R_{\mathrm{Jup}}\right)$ results in the secondary having a slightly higher surface gravity $\left(\log (g)=5.387_{-0.023}^{+0.033}\right.$ dex $)$ than the primary $\left(\log (g)=5.336_{-0.003}^{+0.004}\right.$ dex $)$, despite its lower mass.

\section{Discussion}

\subsection{Substellar Boundary}

The minimum stellar mass for sustained fusion of hydrogen is of interest to understanding stellar interiors, and it is key in determining which objects are viable hosts of habitable planets. This threshold is not a sharp function of mass, as progressively lower mass stars take correspondingly longer times to reach the main sequence. For example, in the BHAC 15 models a $75-M_{\text {Jup }}$ object is clearly a star but takes $\approx 2 \mathrm{Gyr}$ to reach its mainsequence luminosity of $\approx 8 \times 10^{-5} L_{\odot}$. Evolutionary models differ in their detailed predictions of this mass boundary, ranging from $\approx 70 M_{\text {Jup }}$ (the highest mass SM08 object that is clearly substellar) on the low end, 73-75 $M_{\text {Jup }}$ (Chabrier \& Baraffe 2000), 73-79 $M_{\text {Jup }}$ (Burrows et al. 2001, 2011), and as high as 82-83 $M_{\text {Jup }}$ (Fernandes et al. 2019). Models also predict a modest dependence on metallicity for the mass boundary. For instance, according to Fernandes et al. (2019) there is a $\approx 0.04$ dex decrease in the mass boundary per dex of increasing metallicity. Thus, low-metallicity brown dwarfs could be more massive than typical field objects, such as T subdwarfs (e.g., Burningham et al. 2014; Zhang et al. 2019).

One property that is consistently predicted across all models that objects less luminous than $\approx 3 \times 10^{-5} L_{\odot}$ at an age of $\approx 10 \mathrm{Gyr}$ are on a path to fade and cool forever, and some models predict an even higher luminosity for this boundary. The luminosity of WISE J0720-0846B is $(1.5 \pm 0.3) \times$ $10^{-5} L_{\odot}$, well below this conservative, theoretical threshold for a substellar object. Its mass of $66 \pm 4 M_{\mathrm{Jup}}$ is therefore rather high, but not inconsistent with even the lowest modelpredicted mass boundary (Figure 9).

There are relatively few empirical determinations of the mass of the substellar boundary. Dupuy \& Liu (2017) used the first large sample of objects with individual dynamical masses that have luminosities ranging from $\sim 10^{-3}$ to $10^{-5} L_{\odot}$ to determine the substellar boundary as the mass for which objects diverged from a one-to-one relation between mass and $L_{\mathrm{bol}}$. They found a boundary of $\approx 70 M_{\text {Jup }}$ based on a lack of low- $L_{\text {bol }}$ objects at higher masses than that. The uncertainty on this determination is still to be established, but preliminary work from Cancino \& Dupuy (2018) suggests that it is approximately $\pm 4 M_{\text {Jup }}$.

Individual, very cool objects can place a lower limit on the substellar boundary (Table 6). The T1.5 primary in $\epsilon$ Ind BC has a luminosity of $(2.00 \pm 0.08) \times 10^{-5} L_{\odot}($ King et al. 2010) and two distinct mass measurements in the literature. Cardoso (2012) obtained a mass of $68.0 \pm 0.9 M_{\mathrm{Jup}}$ based on relative astrometry from Very Large Telescope (VLT)/NACO and absolute astrometry from VLT/FORS2. In contrast, Dieterich et al. (2018) found a mass of $75.0 \pm 0.8 M_{\text {Jup }}$ by combining their photocenter (unresolved) orbit measured in optical imaging from CTIOPI (Jao et al. 2005) and du Pont/CAPScam (Boss et al. 2009) with a portion of the VLT/NACO relative astrometry also used by Cardoso (2012). Both masses are consistent with the boundary at $70 \pm 4 M_{\text {Jup }}$ from Dupuy \& Liu (2017), even though there is strong tension in the mass results for $\epsilon$ Ind B. We note that Dieterich et al. (2018) report a total mass of $144.5 \pm 1.1 M_{\text {Jup }}$ that relies on both unresolved and resolved astrometry (but independent of the binary's flux ratio), while Cardoso (2012) report $121.2 \pm 1.1 M_{\text {Jup }}$ based on 
Table 6

Dynamical Mass Measurements for T Dwarfs

\begin{tabular}{|c|c|c|c|c|c|c|c|c|c|}
\hline Name & $\begin{array}{l}\text { Spectral } \\
\text { Type }\end{array}$ & $\begin{array}{l}\text { Mass } \\
\left(M_{\text {Jup }}\right)\end{array}$ & $\begin{array}{l}\text { Semimajor } \\
\text { axis (au) }\end{array}$ & Eccentricity & $\begin{array}{c}\text { Mass Ratio } \\
M_{2} / M_{1}\end{array}$ & $\begin{array}{l}\text { Distance } \\
(\mathrm{pc})\end{array}$ & $\begin{array}{c}\log \left(L_{\mathrm{bol}} / L_{\odot}\right) \\
(\mathrm{dex})\end{array}$ & $\begin{array}{l}T_{\text {eff }} \\
(\mathrm{K})\end{array}$ & References \\
\hline HD 4747B & $\mathrm{T} 1 \pm 2$ & $66.2_{-3.0}^{+2.5}$ & $10.1_{-0.5}^{+0.4}$ & $0.735 \pm 0.003$ & $0.077_{-0.005}^{+0.004}$ & $18.79 \pm 0.04$ & $-4.55 \pm 0.08$ & $1380 \pm 50$ & $\mathrm{BJ} 18, \mathrm{Cr} 16, \mathrm{Cr} 18,{ }^{*}$ \\
\hline$\epsilon$ Indi $\mathrm{B}$ & $\mathrm{T} 1 \pm 1$ & $68.0 \pm 0.9$ & $\ldots$ & $\cdots$ & $\ldots$ & $3.6386 \pm 0.0033$ & $-4.699 \pm 0.017$ & $1312 \pm 9$ & $\mathrm{Ki} 10, \mathrm{Ca} 12, \mathrm{BJ} 18,{ }^{*}$ \\
\hline${ }^{* *}$ LUH 16B & $\mathrm{T} 0.5 \pm 1.0$ & $28.55_{-0.25}^{+0.26}$ & $3.557_{-0.023}^{+0.026}$ & $0.343 \pm 0.005$ & $0.8519 \pm 0.0024$ & $1.994 \pm 0.0003$ & $-4.71 \pm 0.10$ & $1190 \pm 60$ & Bu13, Ga17, LS18, * \\
\hline SDSS J1052+4422B & $\mathrm{T} 1.5 \pm 1.0$ & $39.4_{-2.7}^{+2.6}$ & $1.86 \pm 0.03$ & $0.1399_{-0.0023}^{+0.0022}$ & $0.78 \pm 0.07$ & $26.2 \pm 0.4$ & $-4.64 \pm 0.07$ & $1270 \pm 40$ & Du15, DL17 \\
\hline SDSS J0423-0414B & $\mathrm{T} 2.0 \pm 0.5$ & $31.8_{-1.6}^{+1.5}$ & $2.291_{0.0028}^{0.0027}$ & $0.272_{-0.007}^{+0.008}$ & $0.62 \pm 0.04$ & $14.07_{-0.17}^{+0.16}$ & $-4.72 \pm 0.07$ & $1200 \pm 40$ & DL17 \\
\hline DENIS J2252-1730B & $\mathrm{T} 3.5 \pm 0.5$ & $41 \pm 4$ & $1.95 \pm 0.04$ & $0.334 \pm 0.009$ & $0.70_{-0.09}^{+0.08}$ & $15.9 \pm 0.3$ & $-4.76 \pm 0.07$ & $1210 \pm 50$ & DL17 \\
\hline 2MASS J1534-2952A & $\mathrm{T} 4.5 \pm 0.5$ & $51 \pm 5$ & $\ldots$ & $\ldots$ & $\ldots$ & $15.9 \pm 0.3$ & $-4.91 \pm 0.07$ & $1150 \pm 50$ & Li08, DL17 \\
\hline 2MASS J1404-3159B & $\mathrm{T} 5.0 \pm 0.5$ & $55_{-7}^{+6}$ & $3.15_{-0.11}^{+0.09}$ & $0.825 \pm 0.005$ & $0.84 \pm 0.06$ & $23.5 \pm 0.6$ & $-4.87 \pm 0.07$ & $1190 \pm 50$ & DL17 \\
\hline WISE J0720-0846B & $\mathrm{T} 5.5 \pm 0.5$ & $66 \pm 4$ & $2.17 \pm 0.03$ & $0.240_{-0.010}^{+0.009}$ & $0.67_{-0.07}^{+0.06}$ & $6.80_{-0.06}^{+0.05}$ & $-4.82 \pm 0.07$ & $1250 \pm 40$ & Bu15, * \\
\hline 2MASS J1534-2952B & $\mathrm{T} 5.0 \pm 0.5$ & $48 \pm 5$ & $3.40 \pm 0.06$ & $0.0027_{-0.0027}^{+0.0028}$ & $0.95_{-0.16}^{+0.13}$ & $15.9 \pm 0.3$ & $-4.99 \pm 0.07$ & $1100 \pm 50$ & Li08, DL17 \\
\hline$\epsilon$ Indi $\mathrm{C}$ & $\mathrm{T} 6 \pm 1$ & $53.1 \pm 0.3$ & $2.4214 \pm 0.0013$ & $0.5401 \pm 0.0007$ & $0.781 \pm 0.014$ & $3.6386 \pm 0.0033$ & $-5.232 \pm 0.020$ & $975 \pm 11$ & $\mathrm{Ki} 10, \mathrm{Ca} 12, \mathrm{BJ} 18$ \\
\hline Gl 758B & $\mathrm{T} 8 ?$ & $38.1_{-1.5}^{+1.7}$ & $30_{-8}^{+5}$ & $0.40 \pm 0.09$ & $0.048_{-0.015}^{+0.011}$ & $15.603 \pm 0.005$ & $-6.07 \pm 0.03$ & $594 \pm 10$ & Vi16, Bo18, BJ18, Br19 \\
\hline HD $4113 \mathrm{C}$ & $\mathrm{T} 9 ?$ & $66_{-4}^{+5}$ & $23_{-3}^{+4}$ & $0.38_{-0.06}^{+0.08}$ & $0.060_{-0.004}^{+0.005}$ & $41.87 \pm 0.09$ & $-6.0 \pm 0.1$ & $700 \pm 40$ & $\mathrm{BJ} 18, \mathrm{Ch} 18, *$ \\
\hline
\end{tabular}

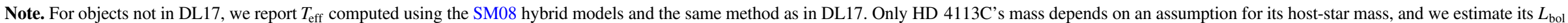
to be the same as UGPS J072227.51-054031.2 (Filippazzo et al. 2015), but with larger uncertainty, given their identical $J$-band absolute magnitudes.

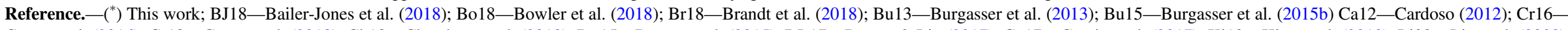

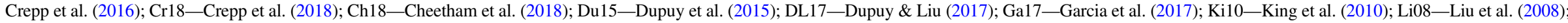
LS18-Lazorenko \& 4Sahlmann (2018); Vi16-Vigan et al. (2016). 
the relative astrometry alone. Almost all orbit parameters have significant tension between the two results, but the only ones directly relevant to the total mass are semimajor axis $(8.3 \%$ higher in Dieterich et al. 2018) and period (3.6\% higher in Dieterich et al. 2018). Given the dependence of $M_{\mathrm{tot}} \propto a^{3} P^{-2}$, this explains the $19 \%$ difference in total mass, and it is mostly driven by the difference in semimajor axis. Cardoso (2012) measured $665.5 \pm 0.6$ mas compared to $721 \pm 8$ mas from Dieterich et al. (2018). It is not obvious what could cause such a large difference in semimajor axis (55 mas, $\approx 4$ NACO pixels) between these two works based on the same relative astrometry data. Systematic errors of $8 \%$ in relative astrometry would defy explanation, especially as the astrometry of Cardoso (2012) was calibrated using images of a wide binary yielding typical precision of 2-3 mas. Likewise, $8 \%$ systematics in the absolute astrometric orbit ( $\sim 15$ mas) seem unlikely, though perhaps somewhat more plausible as the per-epoch precision from Dieterich et al. (2018) was 2-6 mas. Their analysis did not report a goodness-of-fit metric, so we cannot assess whether their errors were appropriate. There is no good explanation for this discrepancy at the moment, but $\epsilon$ Ind B generally lends credence to the existence of massive $\mathrm{T}$ dwarfs in the near-solar metallicity field population, consistent with our results for WISE J0720-0846B.

\subsection{Solar System Encounter}

We have used our new proper motion, parallax, and orbitcorrected system velocity of $82.4 \pm 0.3 \mathrm{~km} \mathrm{~s}^{-1}$ to compute an updated space motion for WISE J0720-0846AB. We find $(U, V, W)=(-57.48 \pm 0.22,-59.18 \pm 0.21,0.22 \pm 0.13) \mathrm{km} \mathrm{s}^{-1}$ and $(X, Y, Z)=(-4.89 \pm 0.04,-4.72 \pm 0.04, \quad 0.271 \pm$ $0.002)$ pc. Mamajek et al. (2015) showed that over timescales of $\sim 100 \mathrm{kyr}$, a linear trajectory is the same within $2.5 \%$ accuracy as compared to more detailed calculations that include the effects of the Galactic potential. Using a linear trajectory, we find that the system's closest passage to the Sun was $0.333 \pm 0.010 \mathrm{pc} \quad(68.7 \pm 2.0 \mathrm{kau})$ at an epoch $80.5 \pm$ 0.7 kya. Our results are in good agreement with the analysis of Mamajek et al. (2015), with our errors being about an order of magnitude smaller thanks to our improved parallax and proper motion measurements. Our results conclusively rule out the hypothesis that WISE J0720-0846AB could have passed through the inner Oort cloud ( $<20 \mathrm{kau}$; Hills 1981) but would have instead passed through the outer Oort cloud where comets can have stable orbits ( $\lesssim 100 \mathrm{kau}$; e.g., Smoluchowski \& Torbett 1984).

\subsection{Comparison to $\mathrm{M}-\mathrm{M}_{\mathrm{K}}-[\mathrm{Fe} / \mathrm{H}]$ Relation}

Mann et al. (2019) have produced the most precise massmagnitude relation to date for $\mathrm{K}$ and $\mathrm{M}$ dwarfs, which can be used to derive masses accurate to $\approx 3 \%$ above the substellar boundary. We computed the mass posterior using their code ${ }^{14}$ and an input 2MASS $K_{S}$-band apparent magnitude of $9.504 \pm 0.020 \mathrm{mag}$ derived from the $K-K_{S}$ color-absolute magnitude relations from Appendix A.1 of Dupuy \& Liu (2017). The Mann et al. (2019) relation gives a mass of $85.0 \pm 2.2 M_{\text {Jup }}$ from just its magnitude and distance, $84.9 \pm 2.3 M_{\text {Jup }}$ if we also provide its metallicity of $[\mathrm{Fe} / \mathrm{H}]=$ $0.15 \pm 0.10 \mathrm{dex}$ (Section 4).

\footnotetext{
${ }^{14}$ https://github.com/awmann/M_-M_K-
}

Our measured mass for WISE J0720-0846A is $99 \pm 6 M_{\text {Jup }}$, which is marginally inconsistent with $(2.1 \sigma$ higher than) the mass derived from the empirical relation. Given that WISE J0720-0846B also appears to be somewhat massive for a mid-T dwarf, this could be a hint that the true total mass is closer to the low end of our posterior distribution. It is also possible that the true mass ratio could be closer to the high end of our posterior distribution, which would shift more of the mass out of the primary and into the secondary, so the secondary would still be relatively massive for its spectral type. Dupuy \& Liu (2017) found a mean mass of $36 M_{\text {Jup }}$ for five T2-T5.5 dwarfs (rms $9 M_{\text {Jup }}$ ), which is expectedly lower than we find for WISE J0720-0846B given the younger mean age of the field population sample used by Dupuy \& Liu (2017). Currently, the dominant source of uncertainty in the total mass is the orbital period $\left(8.06_{-0.25}^{+0.24} \mathrm{yr}\right)$. Given how short this period is, its error should drop rapidly in the next few years, thereby reducing the mass uncertainties and potentially resolving the marginal discrepancy in the primary mass. The mass ratio depends strongly on the photocenter orbit size, and this parameter is still quite covariant with the proper motion of the system, and a longer time baseline will also greatly help in reducing this degeneracy.

\section{Summary}

We present new astrometry and relative photometry from Keck and CFHT, including $K$ - and $L^{\prime}$-band imaging from Keck's new pyramid wavefront sensor. We jointly fit our observations with a thirteen-parameter orbit and parallax solution that yields precise individual dynamical masses and a greatly improved distance measurement. (Like many binaries, WISE J0720-0846 does not have a Gaia DR2 parallax.) The mass of the primary is marginally inconsistent with the empirical mass-magnitude relation and stellar models. The brown dwarf companion is rather massive $\left(66 \pm 4 M_{\mathrm{Jup}}\right)$ compared to other mid-T dwarfs with dynamical masses, which may be partly explained by the current uncertainty in the orbital period and thereby total mass. This mass is consistent with evolutionary models, within the errors, assuming the system is several gigayears old. Such an age is consistent with past interpretation of the stellar host properties and space motion. Finally, our much more precise parallax and proper motion, along with our first accurate orbit determination, enable a more rigorous assessment of the system's recent close encounter with the solar system.

T.J.D. acknowledges research support from Gemini Observatory, which is operated by the Association of Universities for Research in Astronomy, Inc., on behalf of the international Gemini partnership of Argentina, Brazil, Canada, Chile, the Republic of Korea, and the United States of America. M.C.L. acknowledges National Science Foundation (NSF) grant AST1518339. The near-infrared pyramid wavefront sensor is supported by NSF grant AST-1611623. The camera used with the pyramid wavefront sensor is provided by Don Hall with support from the National Science Foundation under grant AST-1106391. M.A.T. acknowledges support from the DOE CSGF through grant DE-SC0019323. Our research has employed NASA ADS; SIMBAD; VizieR; and J. R. A. Davenport's IDL implementation of the cubehelix color scheme (Green 2011). 
Facilities: Keck:II (LGS AO, NIRC2, PyWFS AO), CFHT (WIRCam), IRTF (SpeX), UH:2.2 m (SNIFS).

Software: Spextool (Cushing et al. 2004), emcee (ForemanMackey et al. 2013), M_-M_K- (Mann et al. 2019).

\section{ORCID iDs}

Trent J. Dupuy (i) https://orcid.org/0000-0001-9823-1445 Michael C. Liu (i) https://orcid.org/0000-0003-2232-7664 William M. J. Best (i) https://orcid.org/0000-0003-0562-1511 Andrew W. Mann (ํ) https://orcid.org/0000-0003-3654-1602 Michael A. Tucker (1) https://orcid.org/0000-0002-2471-8442 Gilles Chabrier (i) https://orcid.org/0000-0002-8342-9149 Stanimir A. Metchev (i) https://orcid.org/0000-00033050-8203

Pascal Tremblin (10 https://orcid.org/0000-0001-6172-3403

Aaron Do 지 https://orcid.org/0000-0003-3429-7845

B. J. Shappee (10) https://orcid.org/0000-0003-4631-1149

Dimitri Mawet (10) https://orcid.org/0000-0002-8895-4735

\section{References}

Aldering, G., Adam, G., Antilogus, P., et al. 2002, Proc. SPIE, 4836, 61 Allard, F., Homeier, D., Freytag, B., et al. 2013, MSAIS, 24, 128 Bacon, R., Copin, Y., Monnet, G., et al. 2001, MNRAS, 326, 23 Bailer-Jones, C. A. L. 2018, A\&A, 609, A8

Bailer-Jones, C. A. L., Rybizki, J., Fouesneau, M., Mantelet, G., \& Andrae, R. 2018, AJ, 156, 58

Baraffe, I., Chabrier, G., Barman, T. S., Allard, F., \& Hauschildt, P. H. 2003, A\&A, 402, 701

Baraffe, I., Homeier, D., Allard, F., \& Chabrier, G. 2015, A\&A, 577, A42

Bardalez Gagliuffi, D. C., Burgasser, A. J., Gelino, C. R., et al. 2014, ApJ, 794, 143

Bertin, E., \& Arnouts, S. 1996, A\&AS, 117, 393

Bond, C. Z., Wizinowich, P., Chun, M., et al. 2018, Proc. SPIE, 10703, $107031 Z$

Bonfils, X., Delfosse, X., Udry, S., et al. 2005, A\&A, 442, 635

Boss, A. P., Weinberger, A. J., Anglada-Escudé, G., et al. 2009, PASP, 121,1218

Bouchez, A. H., Le Mignant, D., van Dam, M. A., et al. 2004, Proc. SPIE, 5490, 321

Bowler, B. P., Dupuy, T. J., Endl, M., et al. 2018, AJ, 155, 159

Brandt, T. D., Dupuy, T. J., \& Bowler, B. P. 2018, arXiv:1811.07285

Burgasser, A. J., Gillon, M., Melis, C., et al. 2015a, AJ, 149, 104

Burgasser, A. J., Melis, C., Todd, J., et al. 2015b, AJ, 150, 180

Burgasser, A. J., Sheppard, S. S., \& Luhman, K. L. 2013, ApJ, 772, 129

Burningham, B., Smith, L., Cardoso, C. V., et al. 2014, MNRAS, 440, 359

Burrows, A., Heng, K., \& Nampaisarn, T. 2011, ApJ, 736, 47

Burrows, A., Hubbard, W. B., Lunine, J. I., \& Liebert, J. 2001, RvMP, 73, 719

Buton, C., Copin, Y., Aldering, G., et al. 2013, A\&A, 549, A8

Cancino, A. A., \& Dupuy, T. 2018, AAS Meeting Abstracts, 231, 349.34

Cardoso, C. V. V. 2012, PhD thesis, Univ. Exeter

Chabrier, G., \& Baraffe, I. 2000, ARA\&A, 38, 337

Cheetham, A., Ségransan, D., Peretti, S., et al. 2018, A\&A, 614, A16

Cohen, M., Wheaton, W. A., \& Megeath, S. T. 2003, AJ, 126, 1090

Crepp, J. R., Gonzales, E. J., Bechter, E. B., et al. 2016, ApJ, 831, 136

Crepp, J. R., Principe, D. A., Wolff, S., et al. 2018, ApJ, 853, 192
Cushing, M. C., Vacca, W. D., \& Rayner, J. T. 2004, PASP, 116, 362 Cutri, R. M., Skrutskie, M. F., van Dyk, S., et al. 2003, 2MASS All Sky Catalog of Point Sources (Washington, DC: NASA)

Deacon, N. R., Magnier, E. A., Best, W. M. J., et al. 2017, MNRAS, 468, 3499 de la Fuente Marcos, R., \& de la Fuente Marcos, C. 2018, RNAAS, 2, 30 Dieterich, S. B., Weinberger, A. J., Boss, A. P., et al. 2018, ApJ, 865, 28

Diolaiti, E., Bendinelli, O., Bonaccini, D., et al. 2000, A\&AS, 147, 335

Dupuy, T. J., Kratter, K. M., Kraus, A. L., et al. 2016, ApJ, 817, 80

Dupuy, T. J., \& Liu, M. C. 2012, ApJS, 201, 19

Dupuy, T. J., \& Liu, M. C. 2017, ApJS, 231, 15

Dupuy, T. J., Liu, M. C., Bowler, B. P., et al. 2010, ApJ, 721, 1725

Dupuy, T. J., Liu, M. C., Leggett, S. K., et al. 2015, ApJ, 805, 56

Earl, D. J., \& Deem, M. W. 2005, PCCP, 7, 3910

Evans, D. W., Riello, M., De Angeli, F., et al. 2018, A\&A, 616, A4

Fernandes, C. S., Van Grootel, V., Salmon, S. J. A. J., et al. 2019, ApJ, 879, 94

Filippazzo, J. C., Rice, E. L., Faherty, J., et al. 2015, ApJ, 810, 158

Foreman-Mackey, D., Hogg, D. W., Lang, D., \& Goodman, J. 2013, PASP, 125,306

Gagné, J., Faherty, J. K., Cruz, K. L., et al. 2015, ApJS, 219, 33

Gaidos, E., Mann, A. W., Lépine, S., et al. 2014, MNRAS, 443, 2561

Garcia, E. V., Ammons, S. M., Salama, M., et al. 2017, ApJ, 846, 97

Green, D. A. 2011, BASI, 39, 289, arXiv:1108.5083

Hills, J. G. 1981, AJ, 86, 1730

Ivanov, V. D., Vaisanen, P., Kniazev, A. Y., et al. 2015, A\&A, 574, A64

Jao, W.-C., Henry, T. J., Subasavage, J. P., et al. 2005, AJ, 129, 1954

King, R. R., McCaughrean, M. J., Homeier, D., et al. 2010, A\&A, 510, A99

Lantz, B., Aldering, G., Antilogus, P., et al. 2004, Proc. SPIE, 5249, 146

Lazorenko, P. F., \& Sahlmann, J. 2018, A\&A, 618, A111

Liu, M. C., Dupuy, T. J., \& Allers, K. N. 2016, ApJ, 833, 96

Liu, M. C., Dupuy, T. J., \& Ireland, M. J. 2008, ApJ, 689, 436

Liu, M. C., Dupuy, T. J., \& Leggett, S. K. 2010, ApJ, 722, 311

Maíz Apellániz, J., \& Weiler, M. 2018, A\&A, 619, A180

Mamajek, E. E., Barenfeld, S. A., Ivanov, V. D., et al. 2015, ApJL, 800, L17

Mann, A. W., Deacon, N. R., Gaidos, E., et al. 2014, AJ, 147, 160

Mann, A. W., Dupuy, T., Kraus, A. L., et al. 2019, ApJ, 871, 63

Mann, A. W., Feiden, G. A., Gaidos, E., Boyajian, T., \& von Braun, K. 2015, ApJ, 804, 64

Mawet, D., Bond, C. Z., Delorme, J. R., et al. 2018, Proc. SPIE, 10703, 1070306

Metchev, S. A., Heinze, A., Apai, D., et al. 2015, ApJ, 799, 154

Monet, D. G., et al. 2003, AJ, 125, 984

Puget, P., Stadler, E., Doyon, R., et al. 2004, Proc. SPIE, 5492, 978

Rayner, J. T., Toomey, D. W., Onaka, P. M., et al. 2003, PASP, 115, 362

Robin, A. C., Reylé, C., Derrière, S., \& Picaud, S. 2003, A\&A, 409, 523

Rojas-Ayala, B., Covey, K. R., Muirhead, P. S., \& Lloyd, J. P. 2012, ApJ, 748, 93

Saumon, D., \& Marley, M. S. 2008, ApJ, 689, 1327

Scholz, R. D. 2014, A\&A, 561, A113

Service, M., Lu, J. R., Campbell, R., et al. 2016, PASP, 128, 095004

Simons, D. A., \& Tokunaga, A. 2002, PASP, 114, 169

Skrutskie, M. F., Cutri, R. M., Stiening, R., et al. 2006, AJ, 131, 1163

Smoluchowski, R., \& Torbett, M. 1984, Natur, 311, 38

Tokunaga, A. T., Simons, D. A., \& Vacca, W. D. 2002, PASP, 114, 180 van Dam, M. A., Bouchez, A. H., Le Mignant, D., et al. 2006, PASP, 118, 310

Vigan, A., Bonnefoy, M., Ginski, C., et al. 2016, A\&A, 587, A55

Wizinowich, P. L., Le Mignant, D., Bouchez, A. H., et al. 2006, PASP, 118, 297

Wright, E. L., Eisenhardt, P. R. M., Mainzer, A. K., et al. 2010, AJ, 140, 1868 Yelda, S., Lu, J. R., Ghez, A. M., et al. 2010, ApJ, 725, 331

Zhang, Z. H., Burgasser, A. J., Gálvez-Ortiz, M. C., et al. 2019, MNRAS, 486,1260 